\title{
Métodos estadísticos y funciones SIG: una propuesta de modelado del poblamiento en el ager Tarraconensis
}

\section{Statistical methods and GIS functions: a model proposal of the Tarraconensis ager settlement}

\author{
Ignacio Fiz* \\ Universitat Rovira i Virgili. Institut Català d'Arqueologia Clàssica (ICAC), ERAAUB ${ }^{1}$
}

\begin{abstract}
RESUMEN
En este trabajo se presentan los resultados de la aplicación de determinados métodos estadísticos y funciones SIG sobre los datos conocidos del poblamiento del ager Tarraconensis, es decir, del territorio de la colonia de Tarraco. El estudio está centrado en un periodo cronológico que se extiende desde época ibérica al siglo III d.C., dos momentos en los que se producen sendos cambios en la estructura de la población que ocupa el llamado Camp de Tarragona. Los métodos aplicados nos han permitido modelizar y explicar la estructuración y dinámica del poblamiento. También aportamos nuevos datos e ideas sobre el momento en el cual se produce la llegada de colonos itálicos, cómo se distribuyen en el territorio y cual habría podido ser el estatuto jurídico de la ciudad de Tarraco en el final del siglo II a.C. Por otro lado presentamos modelos explicativos de los periodos de estabilidad en el medio rural durante las dinastías julio-claudia y flavia. Finalmente se analizan y explican aquellos datos que nos muestran cómo dicha estabilidad se quiebra en el siglo II d.C., alterándose por completo la articulación del poblamiento en el territorio en el III d.C.
\end{abstract}

\section{SUMMARY}

This paper presents the results of the application of certain statistical methods and GIS functions on the known data of the ager Tarraconensis settlement, located in the territory of the Tarraco colony. The study focuses on a chronological period covering the Iberian period to the $3^{\text {rd }}$ century AD. The methods used have allowed us to model and explain the structure and dynamics of the settlement. We also provide new data and ideas about the arrival of first italic settlers, how they are distributed throught the territory and what the legal status of the city of Tarraco may have been at the end of the $2^{\text {th }}$ century BC. Additionally, we present explanatory models of the periods of stability in the countryside during the julio-claudian and flavian dynasties.

* A los compañeros de la Universidad Rovira i Virgili, Eva Subías y Joaquín Ruiz de Arbulo, en especial a la primera, por sus correcciones, sugerencias, y recomendaciones.

${ }^{1}$ Equip de Recerca Arqueològica i Arqueomètrica, Universitat de Barcelona (ERAAUB), Grup de Recerca Consolidat de la Generalitat de Catalunya (SGR2009-01173) gracias al apoyo del Comissionat per a Universitats i Recerca del DIUE de la Generalitat de Catalunya.
Finally, we analyze and explain data that shows how this stability was broken in the $2^{\text {th }}$ century $A D$, reversing the articulation of the settlement in the territory in the $3^{\text {rd }}$ century AD.

PALABRAS CLAVES: Ager Tarraconensis, Tarraco, Vecino más Próximo, Análisis de Conglomerados, Kernel Density, Análisis de Cuencas Hidrográficas, SIG.

KEY WORDS: Ager Tarraconensis, Tarraco, Nearest Neighbor, Cluster Analysis, Kernel, Density, Water Bassin Analysis, GIS.

\section{INTRODUCCIÓN}

El presente estudio se ha realizado sobre la base de los datos y conclusiones aportadas por el Proyecto Ager Tarraconensis (PAT) dirigido desde el Institut Català d'Arqueologia Clàssica (ICAC) entre los años 2005 y 2009 y en el que ha participado un amplio abanico de instituciones ${ }^{2}$.

El PAT ha trabajado sobre un área localizada en el llamado Camp de Tarragona, situada en el margen derecho del río Francolí, y con una extensión aproximada de $345 \mathrm{~km}^{2}$ (Fig. 1). El proyecto ha estudiado

\footnotetext{
${ }^{2}$ Los resultados y conclusiones del proyecto PAT (Proyecto Ager Tarraconensis) pueden consultarse en Prevosti y Guitart 2010a; 2010b y en Gorostidi 2010. La justificación y los aspectos metodológicos correspondientes a las prospecciones extensivas y al diseño de las prospecciones intensivas aplicados en este proyecto son desarrollados en Prevosti y Guitart 2010b: 15-27; 37-99. Las prospecciones extensivas consistentes en el estudio y visita de los yacimientos conocidos afectaron a 222 yacimientos que fueron correctamente localizados e incluidos en la base de datos generada. Las prospecciones intensivas, correspondientes al proyecto PAT y al precedente dirigido por Simon Keay (Carreté et alii 1995), se han realizado sobre un $9 \%$ de la superficie total del área de estudio.
} 
este territorio desde una perspectiva pluridisciplinar abarcando una cronología que va desde 500 a.C. a 712 d.C. El ager Tarraconensis se ha analizado desde varias perspectivas como pueda ser un primer estudio del poblamiento, la articulación del territorio, estudios cerámicos, paleoambientales, geológicos y epigráficos.

Nuestro trabajo, aplicado sobre la misma área estudiada por el PAT, profundiza en fórmulas para modelar y analizar el poblamiento en el ager Tarraconensis. Así, la falta de datos arqueológicos completos que permitirían abordar estudios de jerarquización entre asentamientos hace que tratemos de analizar y explotar esta información de forma indirecta utilizando para ello métodos estadísticos y análisis SIG de la información.

\section{EL POBLAMIENTO EN EL AGER TARRACO- NENSIS}

Describimos a continuación cuáles fueron los resultados relativos al poblamiento en el ager Tarraconensis alcanzados por el PAT.

\subsection{Periodo ibÉRICO (SIGLOS IV-III A.C.)}

En nuestra área de estudio se observaron un total de 22 yacimientos para un territorio total de $345 \mathrm{~km}^{2}$. Sin embargo, es necesario destacar que el poblamiento en este momento cronológico presenta un déficit de datos mayor que el cualquiera de las otras áreas costeras de Cataluña (López et alii 2010). La distribución de estos yacimientos, aparentemente dispersa, presenta una nuclearización en torno a una serie de poblados ibéricos (6), y una serie establecimientos rurales (10).

Esta impresión queda confirmada al analizar los datos mediante el estadístico Nearest Neighbor ${ }^{3}$ (Fig. 2). Este proporcionó unos valores de $\mathrm{OMD} / \mathrm{EMD}=1.33$ y Z score $=3.32$, confirmando la estructura de un territorio en el cual los asentamientos se encontraban organizados de forma dispersa. La aplicación del Cost Distance ${ }^{4}$, función calculada mediante SIG, creaba

\footnotetext{
${ }^{3}$ Hay que recordar que Nearest Neighbor o Vecino más Próximo es un tipo de índice que analiza la aleatoriedad en la distribución de un conjunto de localizaciones. Este se basa en comparar la distribución observada, usando para ello la distancia media, con una hipotética distribución aleatoria. Cuanto más próximos a 1 sean los resultados más aleatoria será la distribución de los asentamientos y por tanto menos sujeta a patrones dirigidos de localización. OMD: Distancia Media Observada. EMD: Distancia Media Estimada.

${ }^{4}$ Es el área o superficie que puede recorrerse desde un punto determinado en una distancia temporal o espacial dada. Su cálculo esta basado en los costes energéticos necesarios y pue-
}

unas áreas de explotación que cubrían un amplio sector del territorio pero dejaban a su vez deshabitada una parte importante del área central de la planicie del ager. Ambos datos nos indican una distribución dispersa, pero equilibrada de los poblados y por tanto un control eficiente del territorio. (Fiz 2010a: 278, fig. 6; Prevosti et alii 2010: 376, fig. 3).

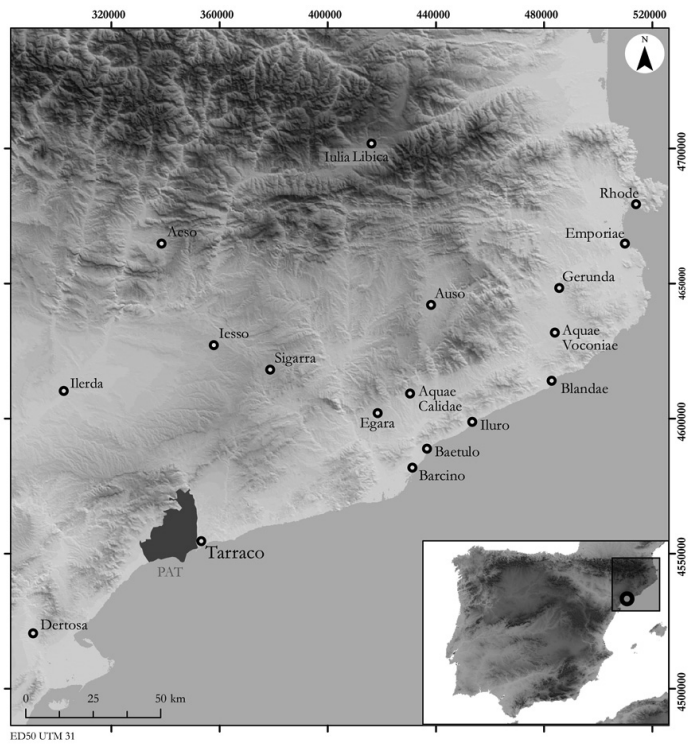

Figura 1. Localización del área del Proyecto Ager Tarraconensis (PAT).

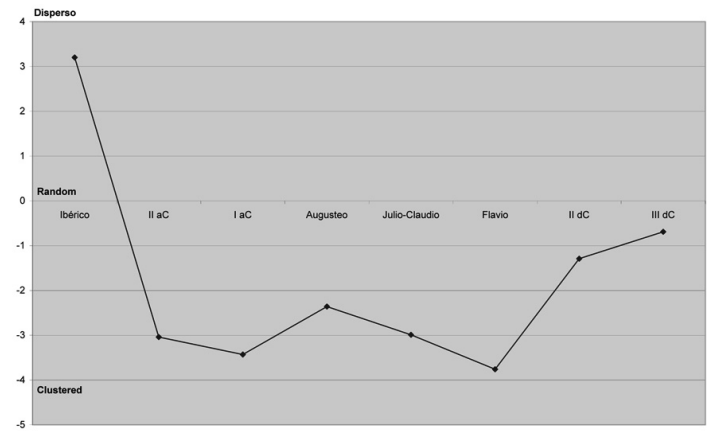

Figura 2. Tabla en la que se indican los diferentes resultados del Vecino más Próximo por periodos cronológicos.

\subsection{Periodo Republicano (Siglos II-I a.C.)}

Con respecto al periodo anterior, destaca en esta fase la gran cantidad de yacimientos documentados

de calcularse gracias a diversas aproximaciones. Generalmente se utiliza para calcular el ACR o AER (Área de captación de recursos o Área de explotación de recursos). Para más información y bibliografía léase Fiz 2010a y 2010b. 
(128), frente a la veintena anterior. Sin embargo una datación más precisa solo ha podido constatar 47 y 64 yacimientos correspondientes a los siglos II y I a.C. respectivamente. Esta multiplicación de asentamientos indica una ruptura con el modelo de poblamiento anterior en el cual el nuevo patrón consiste en una serie de nuevos asentamientos que pivotan en torno a los antiguos poblados. Esta distribución puede deberse a un proceso lento de ruptura con el modelo anterior, caracterizado principalmente por la persistencia durante el siglo II a.C. de determinados poblados ibéricos, 5 de 10, y establecimientos rurales, 6 de 10 (López et alii 2010). Según Arrayás (2004: 38) la transformación no se produce en la primera mitad de siglo II a.C., sino que por el contrario el patrón del periodo ibérico continuaría subsistiendo con el beneplácito de Roma, e incluso potenciando alguno de sus centros; siempre bajo el control de los praesidia romanos. Los cambios se producen principalmente en la segunda mitad del siglo, coincidiendo con un cambio en la política de ocupación romana, basada ahora en el reasentamiento, es decir, en el traslado controlado y dirigido de la población indígena. Este cambio de estrategia, coincidiría con el final de las guerras Celtibéricas, en el 133 a.C., y con la intervención de una comisión senatorial de decemviri agrari ${ }^{5}$ encargada de reorganizar los territorios hispanos. Finalmente (Arrayás 2004: 50-51) afirma que el reasentamiento iría acompañado de una estructura catastral romana (catastro A) como sistema de explotación agraria con una base social principalmente indígena.

Esta hipótesis difiere de la sugerida por otros autores. En primer lugar Francisco Pina Polo (1997: 97 y nota 53; 2009: 224-226) opina que esta comisión de decemviri no habría tomado medidas con respecto a los territorios de la Citerior y la Ulterior previamente conquistados, y que su actuación se habría ceñido estrictamente a los territorios arrebatados a los celtíberos, la Lusitania y la Callaecia. El mismo Pina Polo (1997: 100-101) cita un segundo momento en el que una comisión de decemviri se encontraría presente en la Península entre finales del siglo II a.C.

\footnotetext{
${ }^{5}$ Sabemos por Apiano que era característico de Roma el envío de una comisión de diez senadores para reorganizar los territorios conquistados. Así se conoce: la comisión enviada en el 241 a.C. a Sicilia tras la I guerra púnica; en el 201 a.C. una comisión de legados acompaña a Escipión Africano para acordar la paz con Cartago; en el 167 a.C. tras la derrota del rey Perseo en Pidna, una comisión se encarga de la reorganización de Macedonia e Iliria, y por último citamos, de entre muchos, el caso de Cartago derrotada en el 146 a.C. Esta ciudad no fue devastada hasta que la comisión no deliberó sobre el terreno, decidiendo también la destrucción de las ciudades aliadas de Cartago, así como la concesión de tierra y la definición de estatutos de relación con aquellas ciudades que habían colaborado con Roma (Pina Polo 1997: 85-89).
}

e inicios del siglo I a.C. coincidiendo con un periodo de inestabilidad caracterizado por los conflictos con Lusitanos y Celtíberos, y por la incursión Cimbria del 104 a.C., rechazada por contingentes celtibéricos.

La segunda divergencia a la teoría formulada por Arrayás se deduce de los resultados de los estudios arqueomorfológicos realizados por J. M Palet (Palet 2003; Palet 2008; Palet et alii 2010) según los cuales el territorio del ager Tarraconensis estuvo organizado sobre la base de cuatro tramas catastrales de orientaciones diferentes. Una de estas, Tarraco I, estructuraría la zona central con una orientación y extensión divergente a la detectada por Arrayás, quien además hacía extensiva su propuesta al conjunto de las tres comarcas del Alt y Baix Camp y el Tarragonès (2004: 56-60). Palet también difiere en la datación de la primera intervención sobre dicha trama ortogonal (Palet et alii 2010: 183) que se asigna al final del siglo II a.C., coincidiendo con las reformas urbanas de la Tarraco tardorrepublicana (Macias 2000; Fiz y Macias 2007: 33).

Además de la cuestión cronológica, está el hecho que los datos estadísticos aplicados ya no revelan una dispersión de los asentamientos en el territorio, como así se había registrado para el anterior periodo. Por el contrario, el cálculo del Vecino más Próximo proporciona ahora unos resultados completamente diferentes según los cuales el poblamiento en el siglo II a.C. está claramente agrupado $(\mathrm{OMD} / \mathrm{EMD}=0.77 \mathrm{Z}$ score $=$ -3.04): una tendencia que se acentúa en el siglo I a.C. $(\mathrm{OMD} / \mathrm{EMD}=0.77, \mathrm{Z}$ score $=-3.43)$ coincidiendo con el abandono de los poblados ibéricos.

En el siglo I a.C. se vuelve a producir un incremento en el número de yacimientos con respecto al periodo anterior. Recordemos que durante este siglo se asiste a un programa de asentamiento de veteranos en nuevas colonias y municipios, implicando el control y la administración territorial a través de parcelaciones, también detectadas en diversos estudios (Palahí 2010: 81). César, y después Augusto, impulsa decisivamente esta política con concesiones de estatutos de colonia, acompañados por asentamientos de colonos itálicos en las ciudades peninsulares. En este contexto y tras la concesión del rango de colonia a Tarraco se habría asentado en su territorio, mediante la fórmula de la deductio, un contingente de veteranos entre los años 44 y 37 a.C. (Mar et alii 2010: 298-302)

Finalmente, los estudios arqueomorfológicos antes citados, proponen un gran proyecto de estructuración del ager en el cual se complementaría la trama inicial pre-cesariana del siglo II a.C. con otras dos nuevas, Tarraco II y III. Los incrementos en el número de yacimientos detectados se corresponderían muy seguramente con esta política cesariana. 


\subsection{Periodo altoimperial (siglos I- III D.C.)}

Este periodo se caracteriza también por una reestructuración del sistema agrario, en un proceso de concentración de propiedades (López et alii 2010: 398-399). Una clasificación más precisa por períodos cronológicos constata 74 yacimientos en época augustea, de los cuales 6 son villas y solo una existía en el periodo anterior; 87 en época julio-claudia, siendo 7 de 13 las villas de nueva construcción; 81 con la dinastía Flavia, contando 3 de 15 las villas construidas; 74 en el siglo II d.C., constatando 4 nuevas villas de 19, y finalmente 49 en el siglo III d.C., con una sola villa construida, pero a partir de un establecimiento rural ya existente. Es decir, se produce un aumento continuo de asentamientos hasta época julio-claudia, produciéndose una ligera inflexión en el siglo II d.C. El siglo III d.C. marca una ruptura con el anterior modelo debido a la disminución drástica de asentamientos (López et alii 2010: 402-403). De estos datos se desprende que el cambio de Era significa la reestructuración del modelo tardorrepublicano, con la implantación del sistema de villae durante los siglos I y II d.C., sobre todo a partir de los Julio-Claudios, a costa del abandono de establecimientos rurales.

El estudio estadístico de estos datos mediante el cálculo del Vecino más Próximo apunta a una disposición de los asentamientos concentrada, pero con una tendencia a que las nuevas instalaciones se sitúen de manera más atomizada en el territorio, en especial en época augustea $(\mathrm{OM} / \mathrm{EM}=0.86 \mathrm{Z}$ score $=-2.36)$. A partir de aquí parece producirse una reconcentración de los asentamientos que tendrá su máximo con los flavios $(\mathrm{OM} / \mathrm{EM}=0.79, \mathrm{Z}$ score= - 3.76). Este último dato podría corresponderse con la adopción final del sistema de villae en el siglo I d.C.

Bajo esta dinámica el ager de Tarraco no es un caso excepcional. La cronología de la implantación del modelo de villae en época augustea está registrada arqueológicamente en el nordeste de la Península Ibérica. Aunque se dan casos en los cuales se han detectado estructuras rurales fechadas en las últimas fases del periodo republicano, el consenso general es que en el cambio de era las nuevas construcciones están siguiendo modelos arquitectónicos itálicos, los materiales utilizados responden a artesanos y especialistas itálicos, y la separación entre pars urbana y pars rustica se corresponde claramente con la concepción de las villae (Palahí 2010: 80) y por tanto estamos ante un cambio en el sistema económico y productivo.

La leve disminución de asentamientos en el siglo II d.C. y la drástica reducción en la centuria siguiente son fenómenos paralelos a otras áreas del levante ca- talán, donde encontramos las mismas tendencias con oscilaciones entre el 20 y el $50 \%$ de abandonos para el siglo III d.C., sin que se pueda estimar si estamos ante un despoblamiento o una concentración de la propiedad. (Járrega 2008: 128-132). En efecto, la escasez de yacimientos excavados en su totalidad para este periodo provoca que no puedan definirse patrones de comportamiento concretos (Palahí et alii 2008: 29).

En este contexto del siglo II d.C., el índice Vecino más Próximo pone de manifiesto una alteración: los asentamientos aún parecen seguir un sistema de concentración; sin embargo, mantienen una tendencia a la aleatoriedad $(\mathrm{OM} / \mathrm{EM}=0.92, \mathrm{Z}$ score $=-1.29)$, es decir, no se guían por un patrón de asentamiento concreto. Recordemos que estamos en un momento en el que ya se ha declarado una tendencia al descenso en el número de yacimientos. Esta tendencia se vuelve crítica en el III d.C., cuando desciende drásticamente el número de yacimientos, sean de hábitat o de producción. Los datos del Vecino más Próximo son muy claros al respecto $(\mathrm{OM} / \mathrm{EM}=0.94 \mathrm{ZScore}=-0.69)$, proporcionando una cifra correspondiente a una disposición aleatoria. Evidentemente esto no quiere decir que los asentamientos se hubieran ubicado de forma aleatoria, más aún teniendo en cuenta que todos son continuidad del periodo anterior, sino que son los abandonos los que no obedecen a un patrón concreto, y el resultado es una distribución de asentamientos que adquiere una disposición aleatoria. Entendemos que este "azar" obedece al factor humano, en el cual las razones probablemente estén mucho más ligadas a la compleja situación que está viviendo la transición entre el siglo II y III d.C.

\section{APROXIMACIONES AL ESTUDIO DEL POBLA- MIENTO: METODOLOGÍA.}

Una parte importante de nuestro trabajo en el proyecto PAT fue la adaptación de la base de datos original, Forma Orbis Romani ${ }^{6}$, a nuestras necesidades de explotación de la información. Desarrollada originalmente en Microsoft Office Access, diseñamos diversas consultas en SQL para facilitar su integración como geodatabase de ARCGIS. Una vez creadas estas consultas e incorporadas las tablas en el SIG se pudieron exportar los datos para la creación de gráficos o la realización de estadísticas con paquetes como SPSS v.17.

Sobre la base de todo este trabajo y de la información cuantitativa registrada se pudo realizar el análisis e interpretación de la evolución del poblamiento ${ }^{7}$. A

\footnotetext{
${ }^{6}$ Sobre esta base de datos léase Guitart y Ruestes 2003: 165.

${ }^{7}$ Remitimos a Prevosti et alii 2010.
} 
partir de aquí, y con la utilización de técnicas estadísticas y SIG, nos hemos planteado varias formas de proponer un modelo de comportamiento del poblamiento en el hinterland de Tarraco.

En primer lugar hemos analizado la dinámica poblacional a lo largo de todos los periodos estudiados. Esta dinámica se estudia sobre la base de los yacimientos abandonados, aquellos que son de nueva creación y finalmente los que permanecen. Estos datos nos proporcionan una información interesante acerca de los periodos de actividad constructiva, estabilidad y crisis.

A continuación, y a partir de tres técnicas distintas, se han podido observar las transformaciones producidas en el territorio, y las formas que estas adoptaron complementando y ampliando los datos conocidos hasta el momento. Estas técnicas son: Kernel Analysis, Two Cluster Step Analysis (análisis de conglomerados en dos pasos) y el Análisis de Cuencas Hidrográficas. Las dos primeras utilizan como base de cálculo la distribución puntual en el espacio de los asentamientos sin observar en principio condicionantes geográficos. Sin embargo, la segunda puede estar condicionada a través de las variables utilizadas por aspectos relativos a la relación del yacimiento con su entorno. La tercera, por el contrario, permite la definición de un territorio natural a partir de las cuencas fluviales, sobre el cual se analiza el poblamiento.

\subsection{Kernel Analysis}

La evolución del poblamiento se ha podido apreciar con más claridad gracias al uso sobre SIG del Kernel Analysis (Fiz 2010a: 279-284). Este método permite describir y visualizar la frecuencia de cambio en las observaciones sobre un área concreta. Podemos determinar cuál es la densidad de asentamientos y visualizar al mismo tiempo cuál es la intensidad de un determinado suceso. Como medidas proporcionadas por los SIG encontramos el Kernel Density Estimation introducido en la arqueología por Baxter et alii (1995; más aplicaciones en Hare 2004; Bocquet-Appel et alii 2005; Herzog y Yépez 2010). Es una técnica no paramétrica en la cual se aplica una función de densidad probabilística a cada uno de los objetos de estudio pero dentro de un radio de acción determinado. La superficie resultante es continua y el valor más grande se corresponde con el número de localizaciones dentro del radio escogido. El método, según otros autores (Baxter et alii 1995: 350), es comparable al análisis del Vecino más Próximo siendo a su vez una aproximación informal al análisis de agrupamientos o conglomerados, también llamado Cluster Analysis.

\subsection{Análisis de Conglomerados en dos Pasos}

Entendemos por agrupamiento, conglomerado o clúster, a aquellos elementos de un conjunto global de datos, que tienen una o varias características que los hacen más parecidos o próximos entre sí frente a otros con los que no presentan coincidencias. Por otro lado, el análisis de conglomerados es una herramienta importante para describir, interpretar y explicar los fenómenos relacionados con el poblamiento. La agrupación de yacimientos en una misma área puede ser resultado de diversos factores ambientales, económicos o administrativos. Para determinar cuáles y cuántos son dichos agrupamientos hay diversas técnicas de aproximación ${ }^{8}$. La elegida por nosotros, dentro de los métodos llamados Partitioning Clustering Technique (algoritmos de partición), es el TwoSteps Cluster Analysis (análisis de conglomerados en dos pasos), diseñada para dar una rápida distribución de los agrupamientos. Su gran ventaja es que si desconocemos el número de agrupaciones posibles que podrían generar nuestros datos, el algoritmo busca cuál es el número óptimo.

\subsection{AnÁlisis de Cuencas Hidrográficas}

La arqueología incluye información referente al uso hidrológico del territorio. Por ejemplo, es posible calcular las redes de drenaje de una cuenca hidrográfica y por tanto determinar la relación entre estos y los yacimientos situados en las inmediaciones.

Por definición, una cuenca hidrográfica capta la precipitación, la infiltra y almacena, determinando cómo esta agua es liberada del sistema. También decimos que una cuenca hidrográfica es una región de la superficie terrestre donde las aguas fluyen hacia un mismo punto común, o punto de cierre de la cuenca (llamado Pour Point).

Algunos proyectos de investigación han tomado como base teórica un modelo aproximado a las cuencas hidrográficas planteando la posibilidad de que estas funcionen como espacios de relación social y económica en la idea territorial de un área de captación de recursos, un Cost Distance o la delimitación de un territorio. En este sentido es significativa la aportación de Bintliff (2009: 107) en la definición del concepto de settlement chamber aplicándolo en su estudio del Valle de las Musas: un fértil valle rodeado por tres de sus lados por montañas y capaz de acoger un gran

${ }^{8}$ Para una mejor descripción de los métodos ver Shennan 2004: 216-264; Fletcher y Lock 2005: 140-144; Connolly y Lake 2006: 162-173. 
poblado o una pequeña ciudad y una serie de asentamientos dependientes.

Más interesante aún resulta Torelli, para quien las comunidades individuales de aldeas (vici) surgidas en las áreas del Lacio y Etruria se identificaban con una unidad más amplia (pagi), en virtud de las prácticas religiosas ligadas a la producción agraria pero sobre todo a la utilización de recursos hídricos ${ }^{9}$ (1988: 241-242).

A. Ruiz (2007: 818-822) conceptualiza varias formas de estructura territorial siguiendo este modelo de pagi. El autor plantea diversos casos: un territorio con una fuente hídrica común propia de un único gran oppidum (modelo mononuclear de base pagánica); un territorio polinuclear en el que existe un núcleo de mayor tamaño en la desembocadura del río afluente, y otro situado aguas arriba del pagus (modelo polinuclear de base pagánica); y por último, a nivel de territorio político el que se definiría sobre una base interpagánica.

Siguiendo modelos similares pero desde un aspecto más técnico para el que calcularon las cuencas hidrográficas mediante SIG, encontramos la reconstrucción del sistema de los territorios parroquiales desde la Edad Media en la Turena, Francia (Chareille et alii 2005; Zadora-Rio 2008). En aquel trabajo se puso énfasis en el rol que juega la hidrografía en la delimitación y definición de los territorios comunales, y para esto aportaron como dato fundamental que el 20 $\%$ de los límites comunales coincidían en el territorio de la Turena con algún elemento de la red hidrográfica.

Otro trabajo interesante fue el desarrollado por Löwenborg (2007) sobre los härad o hundare de Suecia, existentes seguramente ya en periodo vikingo, sobre el 1000 d.C. Estas unidades territoriales, conocidas por documentos de finales de los siglos XIII y XIV se dividían a su vez en skiplag (la unidad territorial que proporcionaba un barco al común), orientados quizás a la defensa marítima. Los hundare formaban también distritos judiciales. El autor del trabajo reconstruyó el sistema de captación de aguas y el de las áreas de drenaje o watershed encontrando coincidencias en buena parte de los límites entre los diferentes hundares documentados históricamente y las cuencas naturales.

Pretendemos en nuestro estudio analizar si el comportamiento poblacional responde a una distribución en las proximidades de barrancos y rieras, es decir, si siguen algún criterio sobre la base de las cuencas hidrográficas. Por otro lado, pretendemos utilizar el carácter de unidades de división natural de las cuencas como formas de analizar la localización, dinámica y distribución de la población en el territorio.

\footnotetext{
9 "Pagi sunt qui eadem aqua utuntur" (Festo, p249L) (Tore111 1988: 242, nota 2).
}

\section{RESULTADOS}

\subsection{LA DINÁMICA POBLACIONAL}

Como decíamos, la exploración de los datos desde una perspectiva de asentamientos de nueva creación, abandonos y permanencias puede proporcionarnos una visión de la estabilidad del modelo poblacional a lo largo de un periodo cronológico analizado. Así (Fig. 3), de entre los datos cuantificados relativos a los nuevos asentamientos que no tuvieron existencia en un periodo anterior destacan claramente los fechados en el siglo II a.C., durante el cual tenemos cifras de hasta un $80 \%$ con respecto al número total de yacimientos. Este dato confirma la eclosión de nuevas localizaciones, como ya se comentó anteriormente, marcando el tránsito de la nuclearización hacia la atomización. El siglo I a.C. y el periodo augusteo mantienen valores muy altos en el porcentaje de nuevos asentamientos (cerca del 40\%), datos que podríamos asociar a las llegadas de colonos en el periodo cesariano y augusteo, manteniéndose esta tendencia, ahora reducida a un $20 \%$, en la continuidad dinástica de los julio-claudios. Sin embargo, con la nueva dinastía flavia finaliza este proceso de nueva construcción siendo los porcentajes meramente testimoniales $(3,6 \%)$ y atribuibles a la propia dinámica interna del territorio relativa a la reestructuración hacia el sistema de villae. Estos valores se mantienen a la baja durante el siglo II d.C. $(2,6 \%)$, colapsando por completo en el III d.C.

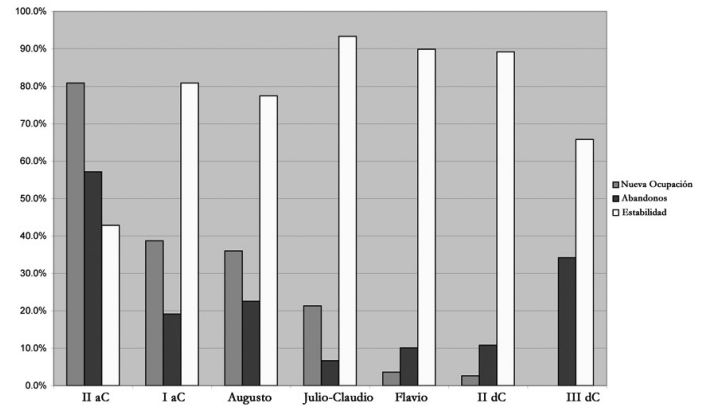

Figura 3. Tabla en la que se indican los yacimientos de nueva creación y abandono, así como los que permanecen, evaluados por periodos.

El proceso de abandono de los asentamientos nos da también informaciones sugerentes. Así, en el siglo II a.C. se había abandonado casi un $60 \%$ de las ocupaciones de origen indígena, lo cual, junto con el alto porcentaje de yacimientos de nueva construcción, nos indica la fuerte alteración que se esta produciendo en el modelo ibérico. El siglo I a.C. y el periodo au- 
gusteo marcan también porcentajes altos en los abandonos $(20 \%)$, que quizás pudiéramos atribuir al final del proceso de aculturación, la adopción del modelo itálico a través de los métodos constructivos, y la reasignación de lotes en los procesos de centuriación del territorio tanto a los habitantes de origen indígena como a aquellos itálicos ya establecidos a lo largo del siglo II a.C. El número de abandonos con respecto al periodo anterior cae al $6 \%$ en época julio-claudia, en lo que finalmente parece una estabilización del modelo. Sin embargo, aquí se produce nuevamente una inflexión y el número de abandonos tiende al alza ya desde época flavia, y continua en el siglo II d.C. Estos datos nos pueden estar hablando de la adopción de las villae como modelo de explotación del territorio, abandonando ahora el anterior de explotaciones individuales. En cambio, los datos del siglo III d.C. señalan que se ha producido el abandono de casi el $35 \%$ de los yacimientos, lo cual, sumado a la nula construcción de nueva obra, sea de hábitat o de producción, nuevamente apunta a un evidente cambio con respecto al modelo anterior.

Observando al tiempo el número de nuevos asentamientos y abandonos nos encontramos que la diferencia resulta positiva desde el siglo II a.C. hasta época julio-claudia. Es decir estamos ante una dinámica de llegadas o de relocalizaciones reflejada en una fuerte actividad constructiva de nueva creación. Este proceso se altera ligeramente en época flavia, seguramente debido a un tímido proceso de reconcentración de propiedades, y se dispara críticamente en el III d.C. (65\%). El último dato que podemos analizar es el de estabilidad, es decir el porcentaje de yacimientos que permanecen con respecto al periodo anterior. Y desde el siglo I a.C. esa estabilidad es superior al $80 \%$, alcanzando el cenit en el periodo julio-claudio (96\%) y hundiéndose en el siglo III d.C. Es decir, se detecta que el modelo se ha asentado en el territorio desde el siglo I a.C., manteniéndose hasta el siglo II d.C.

\subsection{KeRNEL DENSITY ANALYSIS: EVOLUCIÓN DEL POBLA- MIENTO}

El análisis de densidades ha permitido contrastar los cambios producidos en cinco zonas del ager Tarraconensis (Fig. 4). La elección de estas zonas se ha debido a que presentaban aparentemente o una amplia concentración de asentamientos o una alta dinámica de nueva creación y abandono. Se utilizó este método con la intención de observar con mayor detalle el comportamiento que presentaba cada una de las zonas citadas. Estas ocupan un $64 \%$ del territorio estudiado.

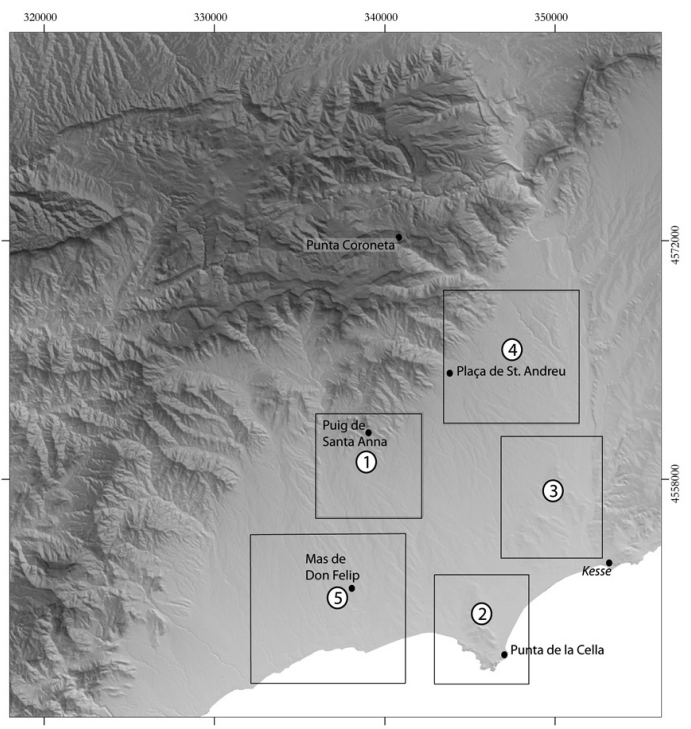

Figura 4. Las cinco zonas seleccionadas para el Kernel Analysis.

La primera zona (Fig. 5), de aproximadamente 22 $\mathrm{km}^{2}$ de superficie, esta situada en el cabo de Salou, y delimitada por las poblaciones actuales de la Pineda,
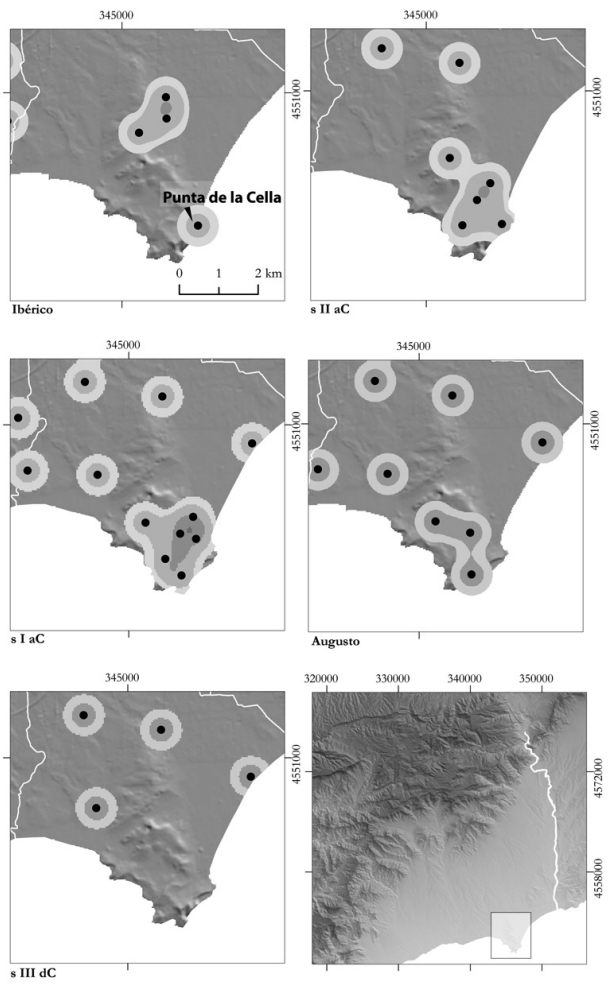

Figura 5. Zona 1. Densidad y evolución del poblamiento entre el siglo IV a.C. - III d.C. 
Vila-seca y Salou. Este cabo se caracteriza por un promontorio, conocido por la Cadeneta, sobre el que se extiende una pequeña planicie llamada el Pla de Maset. Esta área, en la que ya se registraba presencia de poblamiento desde época ibérica, está representada por el poblado ibérico de la Punta de la Cella. El número de yacimientos se incrementa en el siglo II a.C., con tendencia a concentrarse en torno a este núcleo ibérico. En principio la zona no se ve afectada por el proceso de centuriación, alcanzando su máxima densidad de asentamientos en el siglo I a.C., iniciándose aparentemente en época augustea un proceso de despoblamiento materializado en el siglo II d.C.

Una segunda zona de ocupación se había gestado ya (Fig. 6) en época ibérica en torno al poblado del Puig de Santa Anna (Castellvell del Camp). El área, de unos $34 \mathrm{~km}^{2}$, comprende principalmente los municipios de Reus, Riudoms, y les Borges del Camp, afectando a las Rieras de Mas des Sostres, Maspujols y la Alforja. De norte a sur, ocupa el espacio situado a media distancia de la costa, ya en la planicie, hasta los aledaños del primer frente de elevaciones que afectan
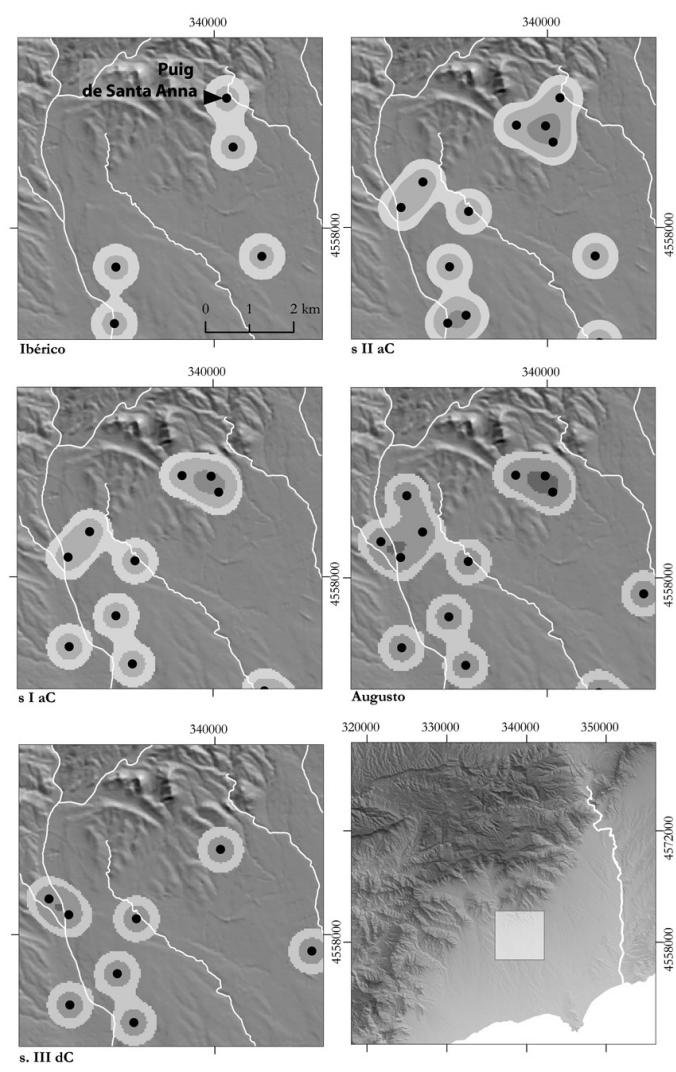

Figura 6. Zona 2. Densidad y evolución del poblamiento entre el siglo IV a.C - III d.C. a la Serra de la Mussara y las Muntanyes de Prades. Para esta ventana de trabajo el análisis de la densidad de poblamiento refleja el incremento en el número yacimientos, fenómeno que se mantiene con Augusto, entrando ligeramente en recesión en el siglo III d.C. Aquí se produce un efecto parecido al de la Punta de la Cella, ya que una parte de las nuevas ocupaciones se realizan cerca del poblado, mientras que el resto se atomiza en la zona. En el siglo I a.C., el abandono del poblado ibérico es paralelo al desplazamiento hacía los cercanos centros de producción cerámica de la Buada, Mas de Gomandí y Mas d'en Corts.

La tercera área estudiada se sitúa a $5 \mathrm{~km}$ al noroeste de Tarraco (Fig. 7). Esta delimitada a oriente por el río Francolí y la via ad Hispanias y comprende los municipios de Constanti (en la parte central), la Canonja, Bonavista , Camp Clar (al sur) y es limítrofe con la Pobla de Mafumet (al norte), afectando una parte de la actual Tarragona. En esta tercera ventana de análisis, que ocupa unos $35 \mathrm{~km}^{2}$, no se registró una ocupación ibérica importante, salvo el oppidum de Kesse. Ya en el siglo II a.C. se produce un gran incremento en el número de asentamientos. La mayor
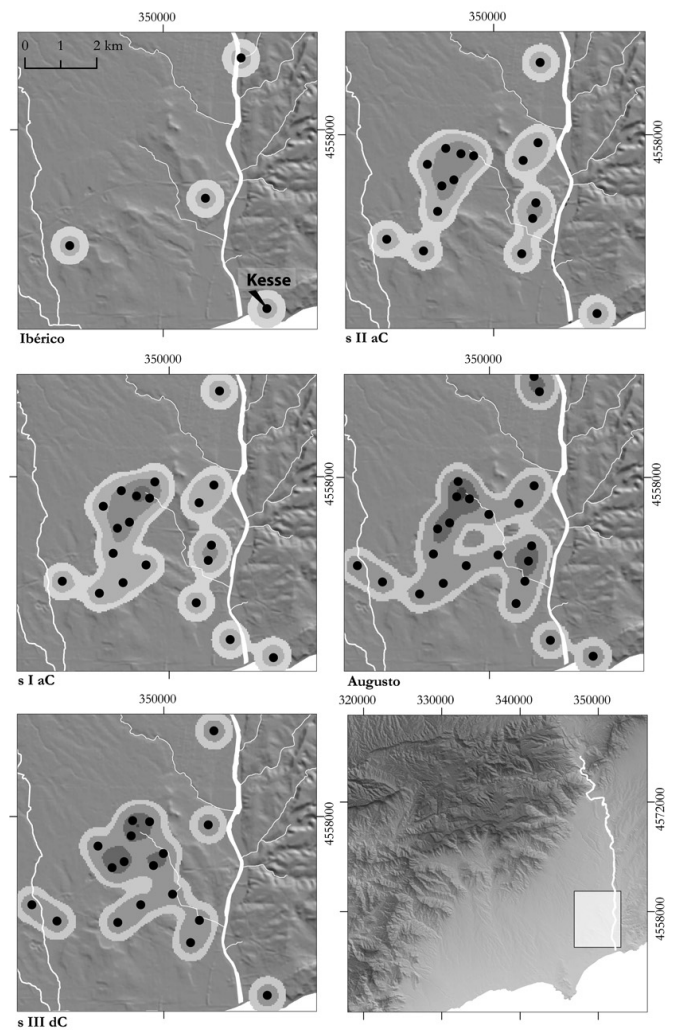

Figura 7. Zona 3. Densidad y evolución del poblamiento entre el siglo IV a.C - III d.C. 
parte de estos se establece en una zona central de la ventana de estudio que coincide con una de las tres tramas centuriadas detectadas en la zona, Tarraco I. Durante el siglo I a.C., y en los periodos augusteo y flavio, este sector experimenta un fuerte incremento. En el siglo III d.C., al igual que las otras dos ventanas estudiadas, también se produce un descenso en el número de asentamientos.

De mayor tamaño $\left(70 \mathrm{~km}^{2}\right)$ es la ventana escogida para representar el margen occidental que se extiende desde el Francolí, hasta los pies de la Sierra de la Mussara (Fig. 8). Situada al norte de la zona analizada previamente comprende los municipios de la Pobla de Mafumet (al sur), la Selva del Camp (sudoeste) Villalonga del Camp (centro) y Alcover (noroeste). El conjunto de rieras incluidas en esta ventana de estudio son (de sur a norte): el torrente de Cassans, la riera de la Selva, el río de la Glorieta, y el barranco de Font Major. En primer lugar cabe tener en consideración que las rieras articulan aquí el poblamiento más que en cualquier otra zona. Esta articulación se mantiene a pesar de las mencionadas operaciones de parcelación
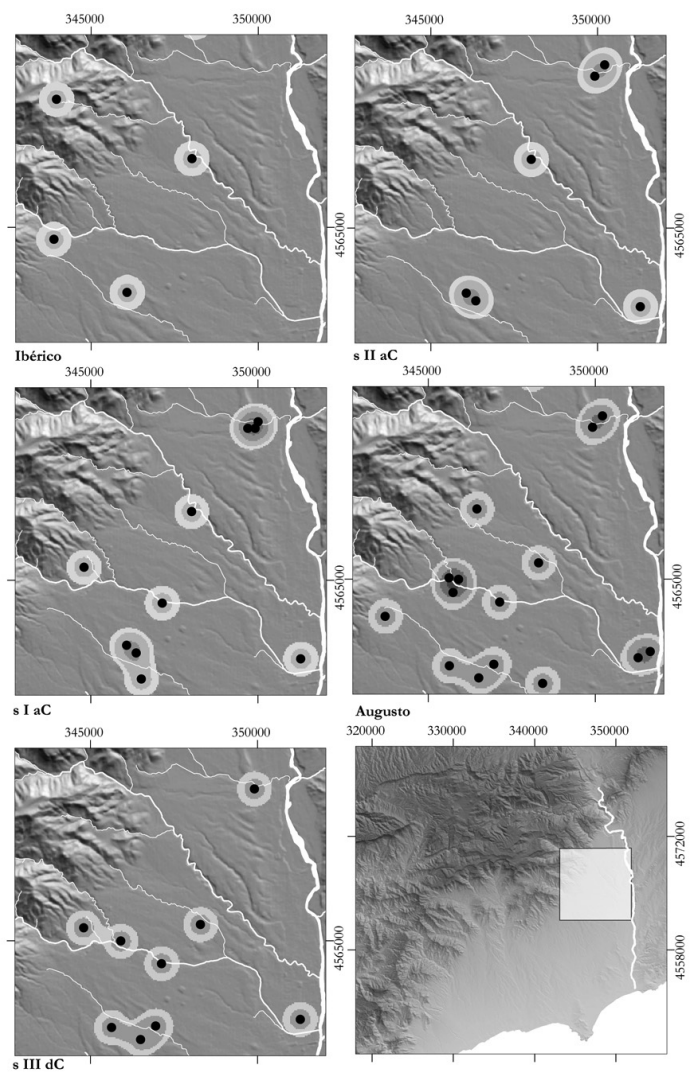

Figura 8: Zona 4. Densidad y evolución del poblamiento entre el siglo IV a.C - III d.C.
(Tarraco III) atribuidas a época cesariana o augustea. Entre el periodo ibérico y el siglo II a.C. se produce un desplazamiento de los asentamientos desde las zonas de pie de montaña a lugares más cercanos al Francolí. Este proceso coincide con el abandono del poblado ibérico de Punta Coroneta, situado más al interior, ya en plena Sierra de la Mussara y el del probable poblado ibérico de Plaça de Sant Andreu (López et alii 2010: 373).

Por último hemos analizado el área costera situada a oriente del cabo de Salou y en principio relacionada con la trama Tarraco I (Fig. 9). Esta ventana, con una superficie de $62 \mathrm{~km}^{2}$, se extiende desde la localidad de Cambrils (al sur) hasta las de Montbrió del Camp y Vinyols (al noroeste). En época ibérica es un área escasamente poblada, destacando por el poblado ibérico de Mas de Don Felip. El siglo II a.C. representa una pequeña expansión hacía la planicie interior y la costa, con una tendencia de los nuevos asentamientos a alejarse del poblado ibérico, del que persiste aún la ocupación. Esta tendencia pudiera obedecer a un binomio centro productor / distribuidor comunicados
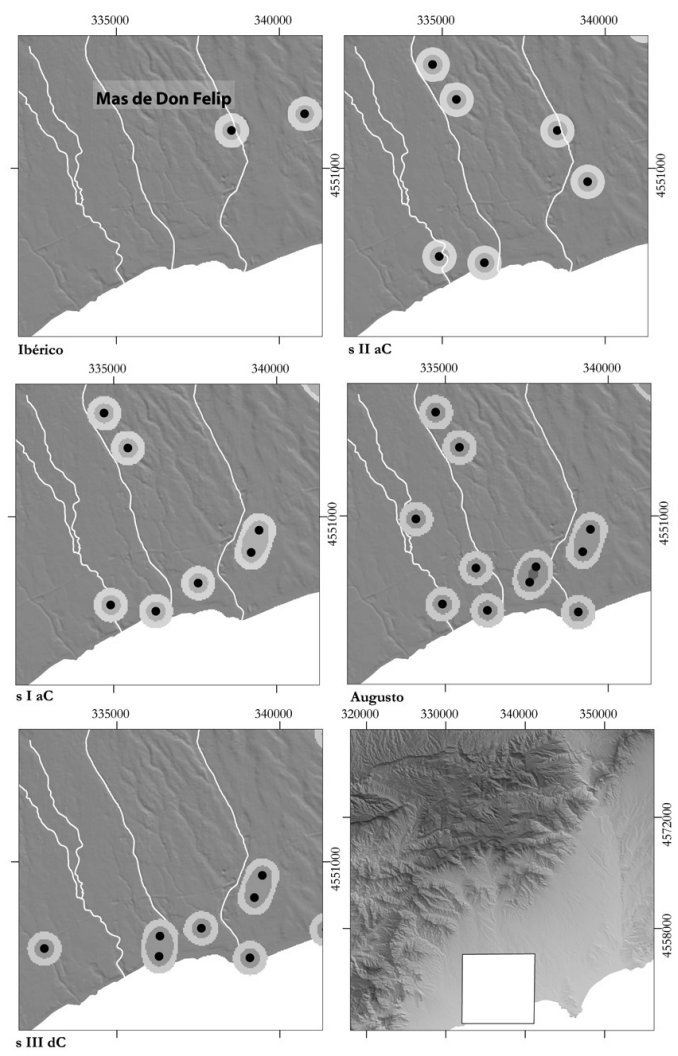

Figura 9: Zona 5 Densidad y evolución del poblamiento entre el siglo IV a.C - III d.C. 
ambos a través del sistema de rieras. Es una dualidad que también se observaba en la ventana del Francolí descrita antes. Sin embargo, la tendencia posterior es la de una mayor densidad de ocupaciones en la costa y en la proximidad de la via Augusta.

Una estadística comparativa entre las cinco zonas estudiadas, donde se analizan el número de yacimientos de cinco periodos cronológicos diferentes, aporta resultados muy interesantes (Fig. 10). En primer lugar observamos homogeneidad en la distribución de las ocupaciones durante la época ibérica, ya que las cinco zonas estudiadas mantienen valores equivalentes. Este equilibrio se altera por completo en el siglo II a.C. en beneficio de la zona 3 (figura 10, columna Kesse) que alcanza un $34 \%$ del total de yacimientos. La causa principal es evidente y es la proximidad del foco catalizador que representa primero Kesse y el praesidium, y después Tarraco. El carácter hegemónico de esta zona como posible centro económico es más que probable a la vista de estos datos. Este carácter será una constante que se mantiene e incluso incrementa porcentualmente en el siglo III, a pesar del descenso generalizado en todas las zonas del número de yacimientos. En el siglo II a.C. se produce además un segundo factor: todas las zonas incrementan el número de yacimientos, pero este crecimiento se concentra solo en las cinco zonas escogidas ya que el resto del área de estudio (un 36\% aproximadamente) no documenta ni un solo yacimiento. Pero, como decíamos antes, solamente la zona 3 (la de Kesse) acumula la mayor parte del hábitat del territorio. El resto de zonas sufren incrementos o decrementos en porcentajes no tan significativos. La zona 2, Puig de Santa Anna, que en época ibérica mantenía un carácter importante en el territorio (22\%), aún lo mantiene $(25 \%)$. Pero este porcentaje desciende ya en el siglo I a.C., en relación al abandono de los poblados ibéricos, donde esta área tenderá a equilibrar su población con el resto, manteniendo su porcentaje hasta incluso el siglo III d.C., momento en que es la segunda zona más habitada $(16 \%)$.

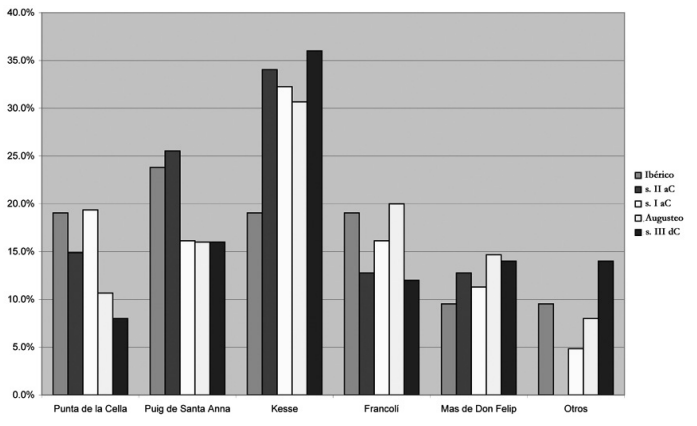

Figura 10. Gráfico de resultados del Kernel Analysis. Porcentaje de yacimientos por área.
El siglo I a.C. y el periodo augusteo son momentos de nueva construcción prácticamente para todas las zonas. La punta de la Cella, zona 1, incrementa el número de yacimientos hasta el siglo I a.C., momento en el cual incluso gana representatividad en el territorio frente a la zona 2, Puig de Santa Anna. A partir de Augusto, comienza un proceso de despoblamiento en el que será la zona menos representativa.

En cuanto al resto del territorio, después de la concentración de yacimientos en las cinco zonas analizadas en el siglo II a.C., se produce un constante aumento que alcanzará al 14\% de los yacimientos documentados en el siglo III d.C. Esto nos indica que progresivamente la ocupación y búsqueda de nuevos espacios fue superando, aunque tímidamente, los límites de las cinco zonas estudiadas. Recordemos que estamos hablando de un $35 \%$ del territorio y de cifras que se mueven entre los 3 y los 7 yacimientos. Esto nos está indicando que un gran porcentaje del territorio permanecía prácticamente despoblado ${ }^{10}$. Los estudios paleoambientales (Riera et alii 2010) indican la existencia de una gran extensión arbórea y una escasa producción agrícola. No sería este el panorama de la zona 3 estudiada, dada la gran densidad de yacimientos existente en un área tan pequeña, pero dichos resultados paleoambientales sí que responden a ese $35 \%$ de territorio prácticamente sin explotar.

La zona 4, Francolí, siendo una de las mayores, solo tiene sus dos momentos de auge en el siglo I a.C. y en el periodo augusteo. En este último es la segunda zona más poblada, en perjuicio de la zona 1, Punta de la Cella, que comienza en ese momento un proceso lento de abandono.

\subsection{AnÁlisis de Cuencas Hidrográficas}

A diferencia del anterior estudio, en que se escogieron las cinco zonas por criterios de densidad de datos, el Analisis de Cuencas Hidrográficas se estructura, como se vio en la explicación teórica, tomando como base la delimitación natural hídrica de las áreas geográficas. La definición de cuencas hidrográficas ha sido posible partiendo de un DEM a 30 metros/ píxel proporcionado por el Institut Cartogràfic de Catalunya. A partir de funciones de GIS ha sido posible la generación de las cuencas hidrográficas (Fig. 11)

${ }^{10}$ Las concentraciones de yacimientos no son estrictamente coincidentes con los transects de prospección ni del proyecto PAT, ni del desarrollado en los noventa por el equipo de Carreté et alii (1995). El número de yacimientos encontrados en estos transects es del $15 \%$ sobre el total, siendo del $9 \%$ el territorio total prospectado en ambos proyectos. 


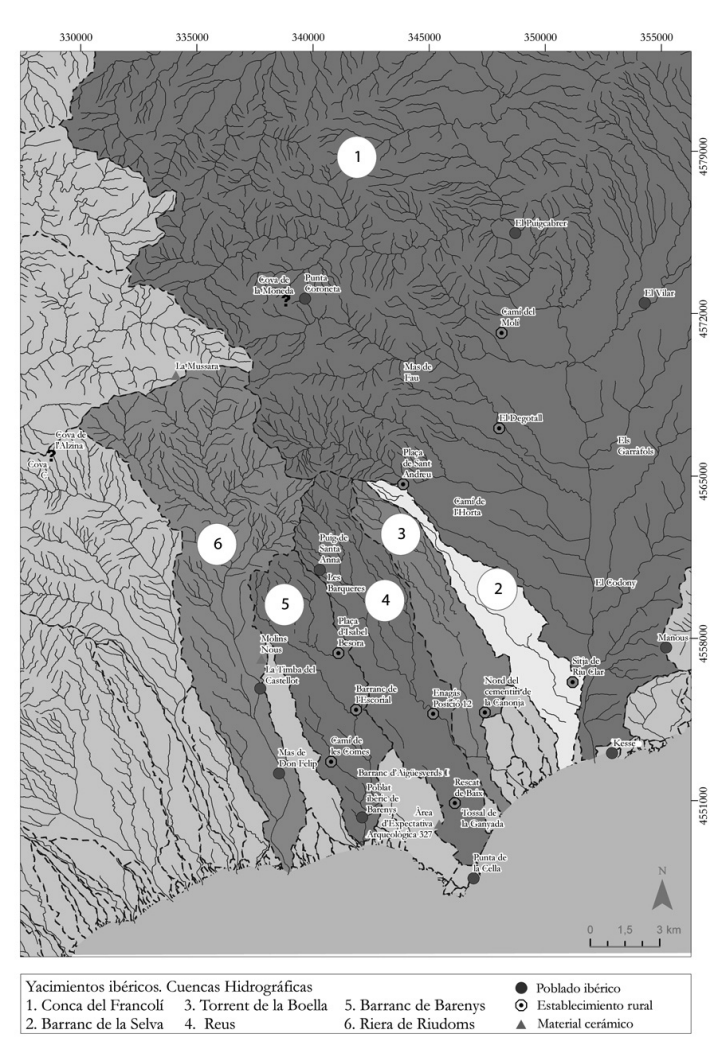

Figura 11. Cuenca Hidrográfica. Distribución de yacimientos en época Ibérica sobre las cuencas hidrográficas.

observando una diferenciación natural entre las rieras del llamado Baix Camp y la cuenca del Francolí, así llamadas por el Departamento de Medio ambiente de la Generalitat de Catalunya. Sobre este mapa se han superpuesto los datos conocidos de los yacimientos de época ibérica los cuales, aparentemente, se adaptan a esta subdivisión natural.

Dentro del sistema de cuencas del Baix Camp destaca en especial el formado por cinco cuencas que acumulan la mayor parte del poblamiento ibérico. Además, el vacío poblacional detectado en el área central mediante Cost Distance, se corresponde aproximadamente con dos cuencas prácticamente deshabitadas, la del Barranco de la Selva y el Torrent de la Boella. En este contexto podríamos identificar el modelo polinuclear de base pagánica como dijimos anteriormente y citado por A. Ruiz. Así por ejemplo, en el poblado de Puig de Santa Anna, situado aguas arriba y limítrofe con las cuencas de Reus y del Barranc de Barenys, encontraríamos dos núcleos situados en las embocaduras de ambas rieras: el poblado ibérico de Barenys y la Punta de la Cella. En la cuenca de la riera de Riudoms esta dualidad estaría representada por Puig de Castellot y Mas de
Don Felip, y finalmente en la cuenca hidrográfica representada por el Francolí: el Vilar y el poblado ibérico en Tarragona (Fig. 11)

Esta situación no parece tener una solución de continuidad en el siglo II a.C. El incremento drástico en el número de yacimientos en el área central del ager Tarraconensis, que ya se detectaba en el análisis de densidades, se concentra ahora en una de las cuencas del Baix Camp, la configurada por el Barranco de la Selva y muy tímidamente en la segunda cuenca deshabitada: el Torrente de la Boella (Fig. 12).

Observando las estadísticas de porcentaje de yacimientos por cuenca entre época ibérica y el siglo II a.C. (Fig. 13), vemos la despoblación de las cuencas del Francolí y el Barranco de Barenys (6 y $8 \%$ respectivamente) frente a otros donde esta tendencia es muy leve (cuenca hidrográfica de Reus de apenas un 2\%). Por el contrario, aquellas zonas que en principio notábamos despobladas en época ibérica, reciben ahora un fuerte impulso. Estas son Barranc de la Selva (un 15\%) y en menor medida el Torrente de la Boella y el Barranco de Riudoms (ambas un 3\%).

Estas tendencias muestran que durante el siglo II a.C. se pudieron producir dos fenómenos. El primero, y en relación a la destrucción o abandono de los poblados del interior, como el Vilar de Valls, pudo ser la desestructuración intencionada del territorio y por tanto el traslado de la población del interior a una zona de planicie, representada por el Barranc de la Selva, próxima al campamento de invierno de Tarraco y por tanto mucho más fácilmente controlable. Por otro lado, los leves incrementos en otras cuencas durante este siglo pueden deberse al lento abandono de los poblados ibéricos cuyas élites locales se mantuvieron fieles a Roma, en un lento proceso de ruptura con la situación anterior. El segundo fenómeno pudiera deberse a un reasentamiento de itálicos a partir de la segunda mitad del siglo.

Estos datos nos indican también que entre el siglo I a.C. y el gobierno de Augusto se produjo un segundo incremento significativo de población, en el área de la cuenca del Francolí y nuevamente en las cuencas del Barranco de la Selva y el Torrente de la Boella. Coincidiendo estos incrementos en zonas articuladas por las tramas Tarraco I y III. Sin embargo, en las zonas del Barranco de Barenys o la Riera de Riudoms, coincidentes con Tarraco II, no se aprecian tales incrementos en porcentaje, pero si incrementos en el número de yacimientos. Este último dato podríamos explicarlo sobre la base de una reorganización más bien de carácter endógeno, es 


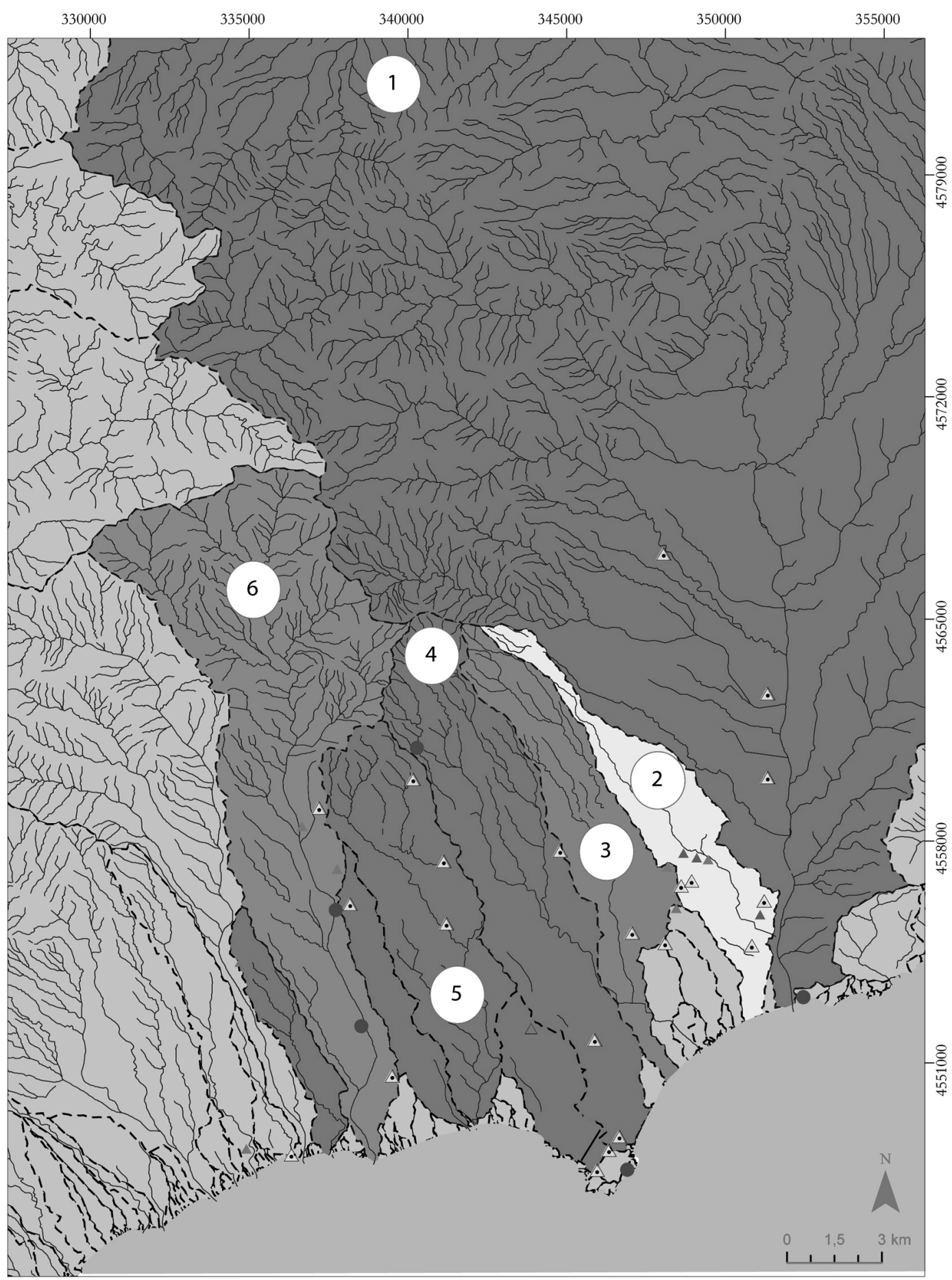

Siglo II aC. Cuencas hidrográficas.

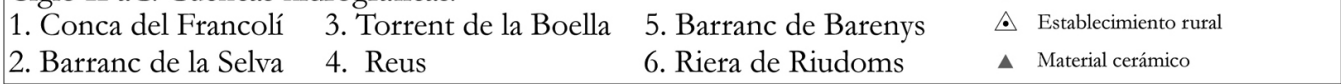

Figura 12. Cuenca Hidrográfica. Distribución de yacimientos en el siglo II a.C. sobre las cuencas hidrográficas. 


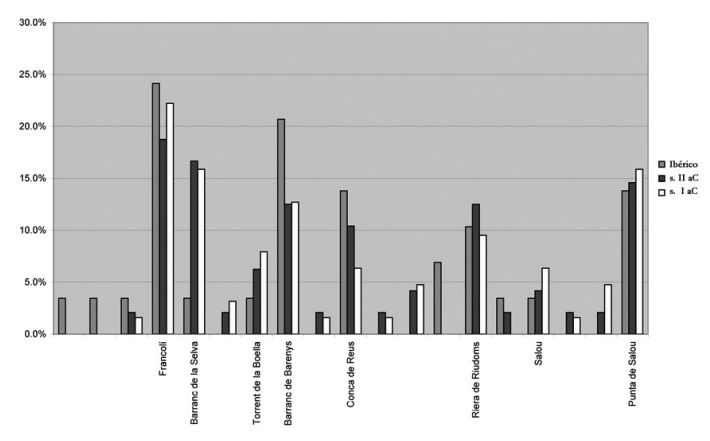

Figura 13. Gráfico de resultados del Analisis de Cuencas Hidrográficas. Periodo ibérico, siglo. II-I a.C.

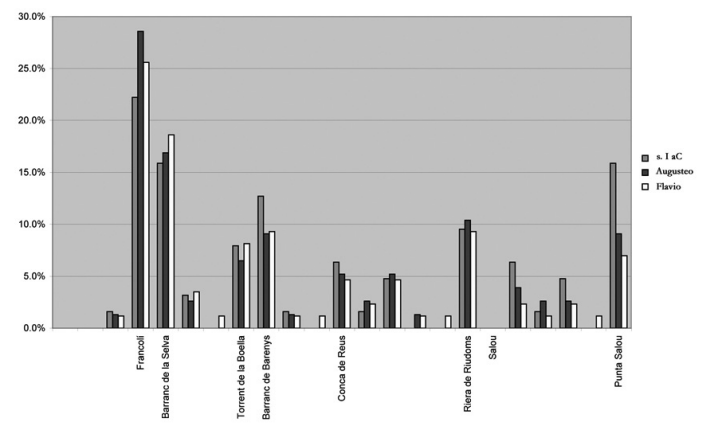

Figura 14. Gráfico de resultados del Analisis de Cuencas Hidrográficas. Siglo I a.C., periodos Augusteo y Flavio.

decir población local a la cual se le ha redistribuido nuevos lotes de tierra.

Entre el siglo final de la república y el periodo augusteo (Fig. 14) encontramos un dato interesante y es que el área de la cuenca del Francolí se repuebla nuevamente de una manera significativa (del 18 y $22 \%$ en el siglo I a.C. y el periodo augusteo).

Por último señalamos el descenso en yacimientos generalizado a todas las cuencas entre los siglos II d.C. y III d.C. Sin embargo, mirando los gráficos (Fig. 15) vemos que tal descenso es común a todas las cuencas, y por tanto es un descenso global a todo el ager. Porcentualmente, tan solo la cuenca del Francolí sufre una perdida destacable (5\%) repartiéndose equilibradamente en otras cuencas.

Como caso aparte hemos unido el conjunto de cuencas que conformarían el cabo de Salou, zona que como ya se vio en los análisis de densidades demostraba una tendencia a la despoblación que se materializaba en el siglo II d.C. Analizado con más detalle esta área que representaba casi el 15\% de los yacimientos desde época ibérica hasta el siglo I a.C., sufre ya en el momento postcesariano una perdida de importancia que descenderá al $6 \%$ en el siglo III d.C.

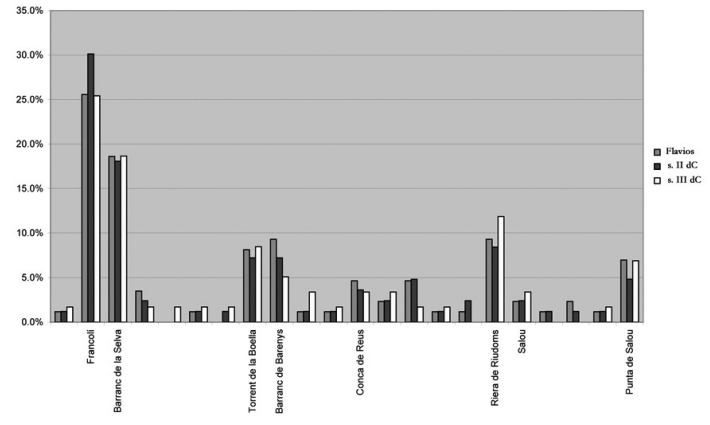

Figura 15. Gráfico de resultados del Analisis de Cuencas Hidrográficas. Periodos flavio, siglo II y III d.C.

\subsection{AnÁlisis de Conglomerados}

La aplicación del Análisis de Conglomerados en dos Pasos como forma de detectar grupos de similar comportamiento nos ha parecido el método más adecuado. En anteriores trabajos, y aplicado a los yacimientos del periodo altoimperial el método había permitido la definición de cuatros grupos (Fiz 2010a: 284-285 y 287, fig. 14), tres de ellos altamente relacionados con las tramas centuriadas detectadas en los estudios arqueomorfológicos, y el último concentrado en el área del cabo de Salou. En aquel momento solo se utilizaron como variables las coordenadas de localización. En el replanteamiento del método y para perfilar mejor los agrupamientos hemos utilizado, aparte de las coordenadas de localización, un conjunto mayor de variables:

- DIST_VIA: Variable que calcula la distancia más corta a la vía de comunicación principal más cercana. En este caso se optó por la via Augusta y la via ad Hispanias (Tarraco-Ilerda) estudiadas por Palet (2008).

- DIST_FR: Distancia más próxima desde el yacimiento al río Francolí.

- DIST_RI: Distancia más próxima a una riera.

- DIST_COD_RI: Código correspondiente a la riera más próxima.

- DIST_LCOST: Distancia más próxima a la línea de costa.

Los valores para cada una de ellas fueron calculados mediante una de las funciones de proximidad que proporciona ARCGIS 9.3. Esta función, llamada NEAR, obtiene la distancia más próxima desde cada uno de los elementos de una capa objeto del análisis a uno o varios recursos representados en otras capas. 
Sobre los datos tabulados y registrados en las cinco variables se aplicó un Análisis de Conglomerados en dos Pasos a los yacimientos, separados por diversas fases cronológicas desde época ibérica al siglo III d.C., utilizando el paquete estadístico SPSS. Los nuevos datos obtenidos, cluster de pertenencia, fueron asignados a cada uno de los ítems sujetos a estudio y reincorporados al SIG para la comprobación de resultados.

\subsection{LA ESTRUCTURACIÓN DEL TERRITORIO}

Los resultados han permitido la creación de una serie de mapas ordenados por cronologías en los que puede observarse la estructuración de los asentamientos en el territorio.

El periodo ibérico presenta cuatro grupos, uno relacionado con el poblado de Tarragona (Fig. 16.1), otro con Punta de la Cella (Fig. 16.2, en el cabo de Salou), el tercero en Puig de Santa Anna (Fig. 16.3) y finalmente el cuarto relacionado con la serie de asentamientos de la cuenca del Francolí (Fig. 16.4). Recordemos que estos cuatro grupos son los núcleos originales, centrados en poblados ibéricos y que este método estadístico nos confirma el carácter intuitivo de las cinco zonas analizadas en el Kernel Analysis. (Fig. 16).

Este panorama se altera en el siglo II a.C. Se mantienen los grupos de Kesse y Punta de la Cella (Fig. 17.1 y 17.2), concentrándose el conjunto de asentamientos del primero en torno al núcleo ibérico y al Praesidium, y situándose también más cerca de lo que posteriormente será la via ad Hispanias. El grupo que en época ibérica había pivotado en torno a Puig de Santa Anna se fracciona en dos. El primero (Fig. 17.3) se mantiene en la órbita del poblado ibérico, pero el segundo (Fig. 17.4) se dispersa desde la costa a pie de montaña. Este fenómeno también se repite en el caso del grupo del Francolí (Fig. 17).

Caso aparte merece un nuevo grupo que aparece en el área central del ager (Fig. 17.6). Recordemos tanto los resultados del Analisis de Cuencas Hidrográficas que indicaban un incremento de asentamientos en cuencas antes casi deshabitadas (Barranco de la Selva y Torrente de la Boella), como del Kernel Analysis, mostrando los aumentos en la densidad de asentamientos en esta área. Ahora el Análisis de Conglomerados nos indica que la creación de este nuevo grupo no surge de los cuatro núcleos originales del periodo ibérico y que no parece producto de la fragmentación o de la desaparición de otro. Como decíamos en el anterior apartado una transformación endógena ha-

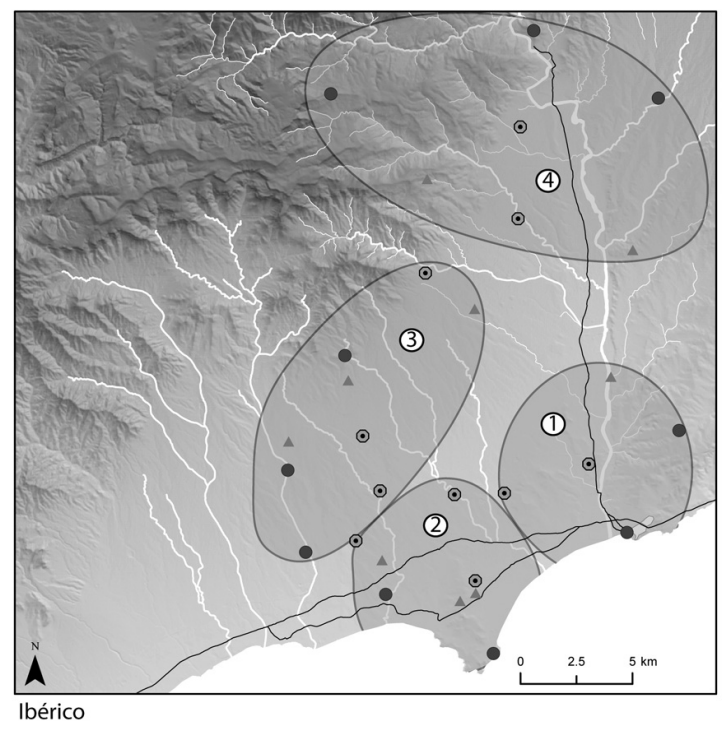

Figura 16. Grupos calculados con el Análisis de Conglomerados en dos Pasos: Época ibérica.

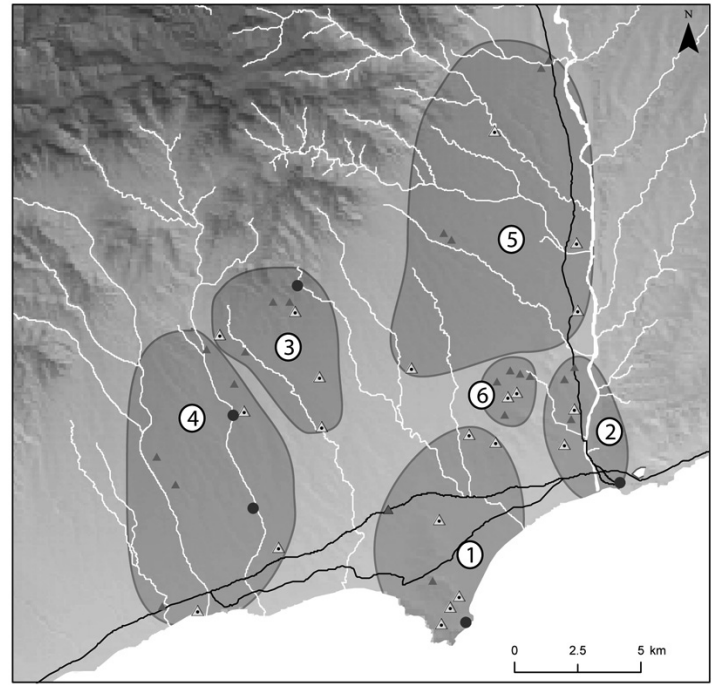

$\mathrm{II} \mathrm{aC}$.

Figura 17. Grupos calculados con el Análisis de Conglomerados en dos Pasos: siglo II a.C.

bría provocado un proceso similar, con porcentajes equilibrados, en todas las zonas donde el núcleo original era un poblado ibérico. Esto no sucedía en el Kernel Analysis con la zona de Kesse (zona 3 ), donde el incremento de yacimientos destacaba con respecto al resto de territorio. El resultado del Análisis de Conglomerados nos indica que efectivamente Kesse crea un grupo de asentamientos que pivotan a su alrededor siguiendo la misma dinámica que otras zonas. Pero al mismo tiempo este cálculo 
estadístico nos informa de que el incremento detectado en el Kernel Analysis corresponde realmente a otro grupo, situado cerca de Kesse pero que no se relaciona directamente con este núcleo urbano. Por último este grupo coincide plenamente con el sector más desarrollado del catastro Tarraco I.

Cabe reconocer que la cronología estudiada representa una horquilla temporal demasiado amplia para determinar en qué momento exacto se produce esta situación. Además, no olvidemos ni la proximidad de esta área de ocupación rural al núcleo ibérico de Kesse y del praesidium romano, ni tampoco el continuo flujo de itálicos en situación de aprovisionar a las tropas estacionadas en la Citerior, así como el carácter portuario de este último emplazamiento sobre el cual convergería la producción económica local, provincial y mediterránea. Por tanto la relación entre este núcleo de ocupación rural, situado en una de las tramas centuriadas, y el desarrollo urbano de lo que será Tarraco creemos es harto evidente.

El siglo I a.C. significa, como habíamos visto, que los poblados ibéricos han sido ya abandonados y por tanto también ha desaparecido el elemento que daba coherencia y organizaba los grupos originales. Aquel que originariamente había conformado el poblado ibérico de la Punta de la Cella queda ahora escindido en dos, uno que ocupa la planicie entre la punta de Salou y Tarraco (Fig. 18.2) y el segundo ocupando el margen oriental del cabo de Salou (Fig. 18.1). El antiguo grupo relacionado con la cuenca del Francolí a su vez se fracciona en uno que ocupa el área central de la cuenca, en relación directa con las rieras dependientes del Francolí, y en otro grupo directamente relacionado con la Via ad Hispanias (Fig. 18.5 y 18.6). El resto de grupos se mantienen pero incrementando el número de asentamientos (Fig. 18).

Podríamos decir que durante el periodo augusteo se produce la aparición de grupos especializados como son los centros de producción cerámica (Fig. 19.5) o aquellos que ocupan la planicie y están próximos a la Via Augusta, y la costa (Fig. 19.3 y 19.4). El grupo central de la cuenca del Francolí (Fig. 19.7) recibe un fuerte incremento en el número de asentamientos (6/11), frente al grupo de la cuenca del Barranco de la Selva que se mantiene casi igual (10/9). Entendemos que los dos primeros se corresponden con las nuevas tramas centuriadas, diseñadas en época cesariana, y por tanto responden a la ubicación de los colonos de la deductio (Fig. 19).

En cambio la fase julio-claudia equivale a la estabilización del modelo de villae que había empezado a gestionarse en la fase anterior. El número de villas prácticamente se duplica (6/13). El grupo que

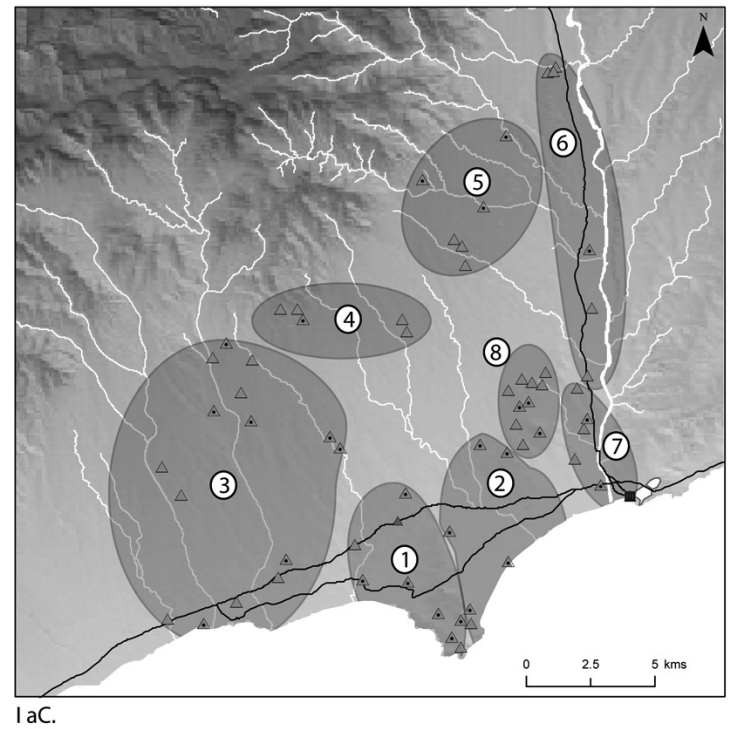

Figura 18. Grupos calculados con el Análisis de Conglomerados en dos Pasos: siglo I a.C.

había caracterizado la parte central de la cuenca del Francolí (Fig. 19.7) se fragmenta en dos (Fig. 20.6 y 20.7) adaptados a los torrentes, barrancos y rieras de esta área: Torrente Cassans, Riera de la Selva, Río de Glorieta y Barranco de la Font major. El grupo que había surgido al norte de Tarraco en la via ad Hispanias (Fig. 19.8) se fracciona a su vez en otros dos grupos (Fig. 20.8 y 20.9). Finalmente, el grupo que caracterizaba la Punta de Salou (Fig. 20.1) encuentra aquí su máxima extensión siendo además el lugar que concentra más villae. Puede resultar paradójico que en el estudio del Kernel Analysis indicáramos que en época augustea se iniciaba un proceso de despoblamiento. En realidad, lo que estábamos viendo es que la zona escogida de análisis, centrada casi exclusivamente en el cabo de Salou y que concentraba los asentamientos en las proximidades del poblado ibérico de la Punta de la Cella se ha comenzado a despoblar y los nuevos asentamientos tienden ahora a ocupar las planicies cercanas estructuradas en las parcelas de las tramas Tarraco I y II (Fig. 20).

El periodo flavio significa, por primera vez, la continuidad del modelo anterior dado que la conformación de grupos no presenta ningún tipo de variación. Recordemos los datos de dinámica poblacional de este periodo en el que se había producido una inflexión ligeramente negativa en la diferencia entre el número de nuevos asentamientos y abandonos.

Sin embargo, los siglos II y III d.C. reflejan la crisis del modelo. En el primer caso la pérdida de solo un diez por ciento de los asentamientos provoca 


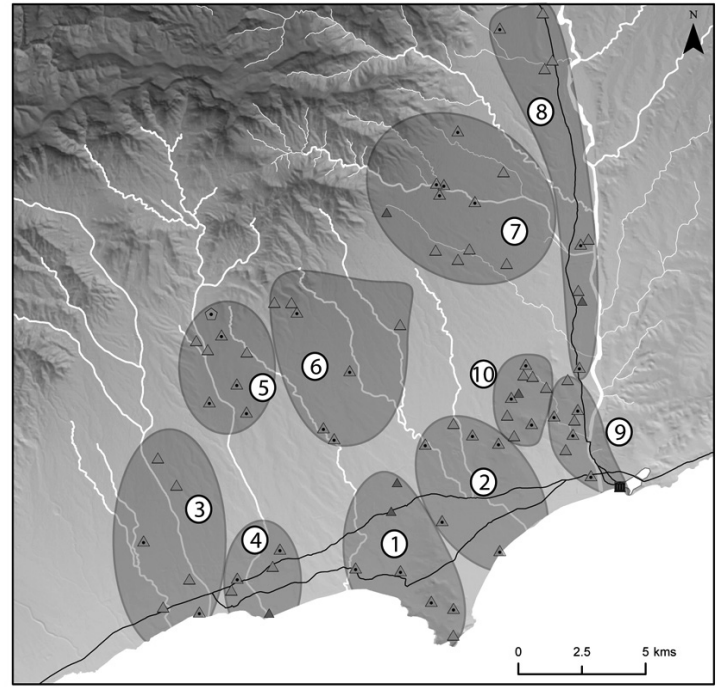

Augusteo

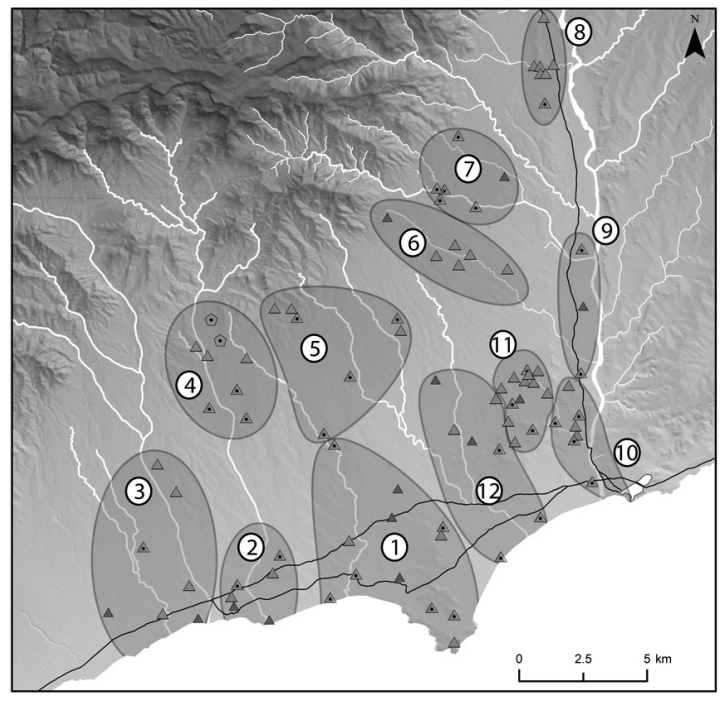

Julio-Claudio

Figuras 19 y 20. Grupos calculados con el Análisis de Conglomerados en dos Pasos: épocas augustea y julio-claudia/flavia.

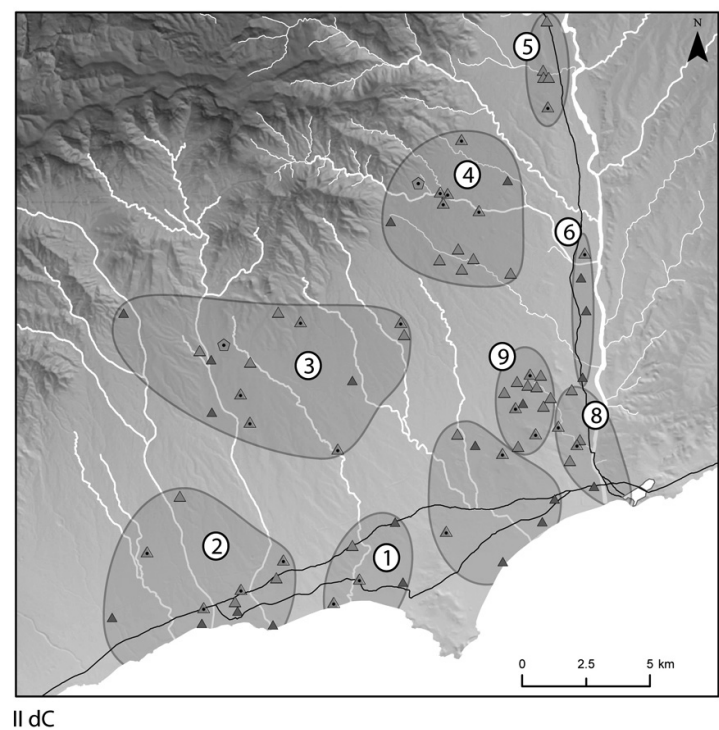

Figura 21. Grupos calculados con el Análisis de Conglomerados en dos Pasos: siglo II d.C.

una fusión en grupos mayores. Una parte importante de las grietas que se producen en el modelo se refleja en el abandono del área de la Punta de Salou y por tanto en el desplazamiento del grupo, orientado ahora por completo hacia la parte oriental del cabo de Salou (Fig. 21.1). Este desplazamiento, provoca un cambio en los dos anteriores grupos ubicados en la línea de costa, que acaban fusionados en uno solo (Fig. 21.2). El siglo III d.C. (Fig. 22) significa la desaparición del grupo que desde

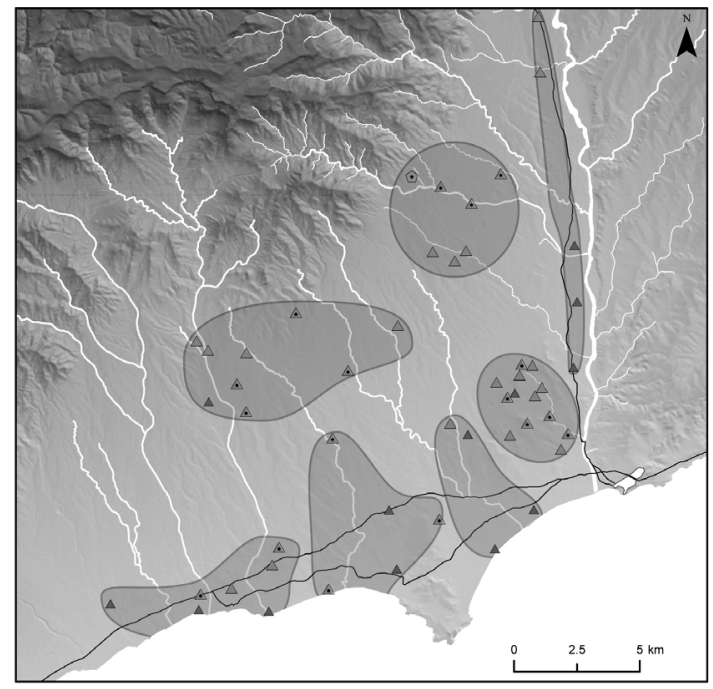

III dC

Figura 22. Grupos calculados con el Análisis de Conglomerados en dos Pasos: siglo III d.C.

el siglo II a.C. había ocupado el espacio cercano a Tarraco, a lo largo del inicio y confluencia de la via Augusta con la via ad Hispanias, provocando por tanto un alejamiento de los hábitats y centros de producción con respecto al centro urbano. Además, aquellos grupos situados cerca de la vía ad Hispanias quedan nuevamente englobados en uno único (Fig. 22). 


\section{CONCLUSIÓN: ALGUNAS APORTACIONES AL CONOCIMIENTO DEL POBLAMIENTO EN EL AGER TARRACONENSIS.}

La diversidad de métodos y análisis utilizados, junto con la comparación con otras áreas del litoral, nos han aportado varias aproximaciones al problema del poblamiento en el ager Tarraconensis. Y la realidad es que partimos de una información muy incompleta, dado que muchos de los yacimientos se encuentran por excavar o no están suficientemente estudiados. Sin embargo, a modo de conclusión creemos que hemos aportado formas exploratorias de análisis del poblamiento e ideas sobre las causas de determinados fenómenos observados en este estudio y en los trabajos previamente realizados.

\subsection{Siglo III-I A.C.: RUPTURAS Y TRANSICIONES}

Hemos visto cómo este periodo está marcado por la permanencia de los poblados indígenas durante el siglo II a.C. y por la eclosión de nuevos asentamientos a lo largo de los dos siglos de estudio. Además, en el análisis de cuencas hidrográficas vemos como aún en el siglo II a.C. se conserva parte de la posible estructura polinuclear de base pagánica en los casos de Puig de Santa Anna-Punta de la Cella y Timba del Castellot-Mas de Don Felip, pero se ha fracturado por completo en el anterior binomio Vilar-Kesse. Sin embargo, los análisis de densidad, Vecino más Proxímo y Análisis de Conglomerados nos matizan que esta gran actividad constructiva no se produjo buscando la máxima ocupación del territorio. Por el contrario, esta eclosión se manifiesta en una concentración dispersa en torno a los centros indígenas hasta entonces reguladores del territorio, manteniendo en apariencia el status quo. Así, retomando la figura resultante del Análisis de Conglomerados del s II a.C. vemos que cuatro de los seis grupos existentes para ese periodo están asociados con poblados ibéricos, mientras que un quinto dependería de la cuenca hidrológica del Francolí, al igual que en el periodo anterior. También veíamos a través del Kernel Analysis que las cinco zonas escogidas representaban en este mismo periodo el $100 \%$ de la obra construida, para un $65 \%$ del territorio redundando aún más en esa concentración aparentemente dispersa visualizada en los valores del Vecino más Próximo.

Solo uno de los seis grupos no parece tener un origen previo. Este (el grupo $14.6 \mathrm{del}$ Análisis de Conglomerados) es el surgido en el área central del ager, la zona 3 del Kernel Analysis, donde también se encuentra la cuenca hidrográfica del Barranco de la Selva no ocupada en época ibérica. Pensamos que este grupo, por la gran diferencia porcentual en yacimientos con respecto al resto, no parece resultado de un movimiento endógeno de población. No parece, vistos estos datos, que las nuevas instalaciones en el área central vayan asociadas a ninguno de los poblados indígenas previamente existentes, ni tan siquiera Kesse que parece tener su propio grupo poblacional cercano a la ciudad.

$\mathrm{Y}$ en relación al periodo del cual estamos hablando, el final del siglo II a.C., es preciso recordar varios datos arqueológicos referentes a Tarraco. En primer lugar, las últimas excavaciones realizadas en el área del foro de la colonia demuestran que en los últimos decenios del siglo II a.C. se había construido un primer templo monumental de tipo itálico, asociado a una plaza cívica. Este edificio estaba organizado en tres cellae, revelando un claro simbolismo capitolino. En el mismo periodo se drenan y canalizan las escorrentías procedentes de un barranco que atravesaba longitudinalmente la ciudad vertiéndolas en el área portuaria a través de una gran cloaca construida con sillares (Mar et alii 2010). Por último, está documentado en este mismo periodo las operaciones de diseño y materialización de una red urbana, incluyendo la plaza cívica y las obras de alcantarillado, de $1 \times 2$ actus de módulo (Macias 2002). Estas reformas más propias de un núcleo ex-novo, serían, en principio, producto de la expansión de la ciudad indígena libre y federada de Kesse/ Tarraco (Mar et alii 2010: 258-260). Es decir, todo el proceso de construcción urbana iría asociado a la expansión del viejo núcleo ibérico, favorecido por la llegada de los itálicos, publicani y negotiatores, asociados al suministro del praesidium y también como gestores del centro de importación y exportación de productos que debió significar Tarraco en ese momento.

Pero reformulando estos datos y observando el problema ahora desde una perspectiva territorial pensamos que en algún momento, a lo largo del siglo II a.C., parte del territorio sujeto a este estudio tuvo que pasar a formar parte del ager publicus. Según Tito Livio las cantidades de tierras confiscadas podían ser de 1/3, 2/3 o la mitad (Moatti 1993: 83). Hemos visto como el área central del ager, y en concreto el Barranco de la Selva, está prácticamente deshabitada durante el periodo ibérico, siendo paradójicamente la que recibe el mayor porcentaje de nuevos asentamientos en el siglo II a.C., siguiendo los modelos de estructuración en pagi citados por M. Torelli y A. Ruiz. Teniendo en cuenta que los estudios arqueomorfológicos han 
demostrado la articulación del ager Tarraconensis sobre la base de tres tramas ortogonales, podemos aventurar que la parte central, en origen pre-cesariana, se correspondería con ese tercio aproximado del total del territorio. En este caso Roma habría ejercido su política de ocupación pero respetando, en principio y a la vista de estos datos, la distribución original en el territorio indígena, situándose en un punto del territorio que no podía provocar conflictos con el resto de comunidades locales, pero organizándose dentro de una unidad geográfica natural como es una cuenca hidrográfica, al modo de los pagi.

Es decir, este ager publicus, se convirtió en un espacio susceptible de ser repartido cuando así fuera requerido. Sin embargo no hay una posibilidad de deductio, pues el cambio de estatuto jurídico y el asentamiento de colonos se producen tras la muerte de César. Por tanto, ¿bajo qué fórmula podía realizarse una repartición de lotes entre "colonos" itálicos sin que fuera necesaria la fundación de una colonia o un municipium?

Sabemos que la adsignatio viritim era una fórmula escogida para un reparto de tierras de forma individual utilizada cuando la asignación no iba acompañada de una deductio y por tanto de la necesidad de la existencia de una colonia o municipium próximo donde inscribirse. Son bastantes los casos itálicos en los cuales se realiza este tipo de asignación: Ager Picenus et Gallicus (232 a.C.), a lo largo de la Via Aemilia, en el Ager Falernus y cerca de los Forum Popilii y del Forum Claudii (Crespo 2009: 289-290).

Es decir, en el contexto urbano se acometieron una serie de obras que implicaron la creación de un espacio cívico, comercial y religioso, mientras que por otro lado se estructuró el territorio y se adoptó una fórmula de asignación de lotes de tierra a itálicos, pues no pensamos que pueda ser otro el origen del incremento de asentamientos en el área central del ager. Ambos procesos pudieron producirse en un mismo espacio de tiempo, al final del siglo II a.C. Además, una comisión de decemviri se encontraba en ese mismo periodo en la Citerior encargada de reorganizar la provincia. Se podría argumentar que una comisión de decemviri solo actuaba cuando se producía un proceso de conquista de nuevos territorios. Pero los sucesos acaecidos en Sicilia provocados por la primera guerra servil en el 132 a.C. obligaron a que una comisión fuera nombrada para acompañar al cónsul Rupilio tras sofocar la revuelta, encargándose de la elaboración de la lex Rupilia, redactada para la reorganización del territorio de la isla. Ante este precedente, no es descartable que la segunda comisión llegada a Hispania asumiera la reorganización de la provincia Citerior y por tanto la organización y estructuración territorial y urbana de Tarraco.

Ahora bien, si Tarraco no podía ser una colonia, ya que este cambio se produce, como se dijo, con posterioridad a la muerte de César, ¿qué otra fórmula jurídica podía permitir la gestión conjunta de una realidad urbana y un territorio centuriado, ambos con posibles acentos itálicos?

Actualmente algunas líneas de investigación han propuesto que bajo la fórmula de los fora se organizó la emigración romano-itálica en la Península (Crespo 2009: 289). En Cataluña encontramos por ejemplo el caso del poblado ibero-romano de Can Fatjó (Rubí), identificado como un vicus pero también como un fórum sobre la base de la existencia de una posible zona monumental. Un segundo caso habría sido el del municipium Segarrensis, que probablemente habría funcionado como un forum destinado a las diversas actividades relacionadas con la ganadería, pues su situación está entre la depresión prelitoral y la ruta de la sal dentro de las rutas de trashumancia horizontales de Cataluña (Prieto 2002: 154-156).

Esta fórmula jurídica podría haber sido aplicada a Tarraco dado que un forum equivale a un tipo de comunidad urbana asentada allá donde se había realizado una adsignatio viritana, sirviendo por tanto de referencia a los emigrantes instalados en el territorio. Los municipium, colonia, praefectura, forum y conciliabulum, mantenían características comunes entre sí, como la de gozar de cierta autonomía o la de escoger sus propios magistrados y consejo local. La existencia en la Tarraco tardorrepublicana de magistrados está confirmada por dos epígrafes que aluden a dos libertos que ejercen de magistri de la comunidad republicana de Tarraco (Alföldy 1991: 31 RIT 5). En este sentido consideramos la existencia de una bipolis, la primera de las cuales sería esta Tarraco posiblemente organizada como forum y en segundo lugar el antiguo oppidum ibérico, fuera de la estructura legal de Roma, organizada como foederata o libera et immunes.

Además, las asignaciones viritanas también permitían la obtención de lotes de vivienda urbana (Moatti 1993: 40, nota 37), por lo que podríamos aventurar que la ciudad creada en Tarraco, diseñada sobre la base de parcelas, serviría a su vez de vivienda a los individuos receptores de la asignación viritana.

No olvidemos que los fora podían ser sede de una prefectura, es decir de una circunscripción jurídica a la cual podían recurrir las comunidades itálicas en caso de conflictos judiciales. Y evidentemente, los fora eran además espacios de desarrollo de las actividades comerciales y en este sentido el binomio Kesse/Tarra- 
co ofrecía el contexto geográfico y político adecuado para este uso. Finalmente, la importancia adquirida por algunos de estos fora estaría confirmada porque en un segundo momento se convertirán en una colonia, como en el caso de Forum Iulii, la actual Fréjus (Gross y Torelli 2007: 278-279).

En este sentido, y a raíz de la reunión de provinciales que convoca César en Tarraco, se dan las condiciones para dar un paso más allá y promocionar el estatuto jurídico de la ciudad, acompañado a su vez por un segundo proceso de estructuración del territorio y asentando, ahora bajo la fórmula de la deductio, un contingente de veteranos ya asociados a las clientelas de César. Este sería el segundo proceso de ordenación del territorio contrastado arqueomorfológicamente. Esta creación de nuevos asentamientos lo hemos visto en los datos aportados en la tabla 2, continuará en época augustea y julio-claudia.

\subsection{La estabilidad: Augusto y el Siglo I D.C.}

La eclosión de asentamientos individuales, y la atomización del poblamiento tienen su máximo exponente en época augustea, aunque los análisis mediante Vecino más Próximo detectan una tendencia a la concentración de asentamientos en zonas concretas. El Análisis de Conglomerados evidencia una tendencia a la formación o concentración de grupos en relación a la implantación de las villae como modelo económico preponderante.

El estudio de la dinámica poblacional y el Análisis de Conglomerados proporcionan datos cuantitativos que expresan un panorama de equilibrio y estabilidad a lo largo del siglo I a.C. En el primero los porcentajes de nueva obra construida y los abandonos indican una dinámica interna posiblemente relacionada con la implantación del modelo de las villae. La actividad constructiva aún era del $20 \%$ en época julio-claudia y del $5 \%$ en el periodo flavio. Y también hemos visto como la diferencia nueva construcción/abandonos se invierte en época flavia por primera vez. Por otro lado, la estructura de grupos definida ya en época julioclaudia se mantiene idéntica en el siguiente momento cronológico. Por tanto entendemos que es en el periodo flavio cuando el modelo itálico de romanización ha alcanzado su máximo apogeo y el sistema de explotación de las villae está en pleno funcionamiento. Los factores decisivos en esta estabilidad han de ser la política favorable de la nueva dinastía con respecto a Hispania, así como las reformas urbanas realizadas en la parte alta de la ciudad en beneficio de la consolidación de Tarraco como sede del Concilium Provinciae.

\subsection{LA QUIEBRA DEL SISTEMA: LOS SIGLOS II Y III D.C.}

El Análisis de Conglomerados aplicado al siglo II d.C., pero en especial al siglo III, evidencian que el modelo estructural del territorio se había quebrado. Estamos ante un proceso en el que se están abandonando una gran parte de las propiedades individuales y la reconcentración y reforma de las explotaciones rurales relativas a los fundi. La nula dinámica constructiva de nueva creación nos esta indicando que la iniciativa individual ha dejado paso a un proceso económico en el cual las diferencias entre las diversas capas sociales se han tornado críticas por perdida y acumulación en pocas manos de la propiedad. El carácter aleatorio de los abandonos, visto en el Vecino más Próximo, nos indica que nos encontramos seguramente ante factores humanos, y por tanto no dependientes de patrones concretos. La evidencia de que los abandonos resultan prácticamente comunes a todos las cuencas hidrográficas del territorio señala que este cambio responde a factores que no dependen de políticas concretas dirigidas a colonizar o despoblar un territorio, como se viera en el siglo II y I a.C. Solo podemos pensar que los abandonos forman parte de un proceso de reconcentración, y por tanto a la pérdida adquisitiva y patrimonial de un amplio sector social, sin olvidar, los efectos que hubieran tenido sobre la población las represalias a que Septimio Severo sometió a los partidarios hispanos de Clodio Albino.

Otro dato a tener en cuenta para el siglo III es el "alejamiento" de los yacimientos con respecto a la ciudad. Es un alejamiento manifestado en el abandono de los lugares más cercanos a Tarraco y por tanto redunda en una sensación de distancia. En este sentido recordemos que podían ejercer magistraturas aquellos que tuvieran sus propiedades a una distancia máxima de una milla romana (1481 metros) de la colonia (Melchor 2009: 394). La villa romana de Ceratonia, situada a una distancia próxima a la citada y seguramente propiedad de los Apronii ${ }^{11}$, fue abandonada durante este periodo. Este fenómeno podía relacionarse con la incapacidad de las familias, hasta entonces principales, de seguir ejerciendo de evergetas y de ser el soporte económico de la deficitaria política urbana.

Otra explicación a estos datos, sobretodo del siglo II d.C., podría venir de la llamada "extenuación hispana" citada en la Historia Augusta. Esta surgía por un lado de la imposibilidad de los ricos ciudadanos de hacer frente a las cargas de ocupar las magistraturas urbanas, situación acentuada para aquellos que

\footnotetext{
${ }^{11}$ Miembros del orden senatorial o ecuestre pertenecieron a la gens de los Apronii, ostentando cargos como legatus iuridicus o II vir quinquennalis. (Gorostidi et alii en prensa).
} 
accedían al orden senatorial, quienes se veían en la tesitura de evadir sus fortunas, siguiendo las directivas de Trajano, invirtiendo de forma obligada en el entorno de Roma (Mar y Ruiz de Arbulo 2011: 521). Es decir, los abandonos y la nula actividad constructiva implicarían, hablando en términos actuales, una forma de deslocalización y fuga de capital, de la propiedad y la producción. Sin embargo, las acciones para atenuar los efectos desestabilizadores en la economía hispana de esta política imperial no se aplican hasta la década del 160-170 d.C., bajo el mandato de Marco Aurelio. Esta explicación contextualizaría los resultados vistos para el siglo II, en el que se agrieta el modelo territorial del siglo anterior, y podría dar explicación a los dramáticos datos del siglo III d.C. Recordemos que en este periodo solo está registrada la construcción de una única villa, que además es resultado de la reforma de un establecimiento rural precedente.

Nos hemos planteado una tercera posibilidad, la cual justificaría en cierta medida el carácter "aleatorio" que habíamos observado en el estudio del Vecino más Próximo. Es decir, la aparición de un patrón no controlado, o como decíamos en su momento la presencia del "factor humano" que provocaría el abandono generalizado visualizado en los datos obtenidos para todas las zonas estudiadas en el Kernel Density, el análisis de las cuencas hidrográficas y en el Análisis de Conglomerados. A falta de cronologías de más detalle que nos permitirían definir si esta "crisis" en el mundo rural se produce de forma escalonada y gradual o súbitamente nos hemos planteado la posibilidad de que dicho factor fuera en realidad los efectos provocados por la pandemia de peste del siglo III. Esta se produce entre los mandatos de Decio (249-251) y Trebonio Gallo (251-253), teniendo su brote original en Egipto en el 251 y, en principio, las fuentes no son explícitas, afectando a todo el Imperio Romano, en especial a su ejército. La epidemia tuvo un rebrote en el 270 y provocó la muerte del propio emperador Claudio el Gotico ${ }^{12}$. Como decimos, no es más que

${ }^{12}$ (Zosimo I, 26,37 y 46). Por otro lado sabemos que en el siglo II también se produjo la llamada peste de los Antoninos. Originada en Etiopía, llegó hasta Seleucia en el 164, durante la campaña militar de Lucio Vero contra los partos en Armenia y Mesopotamia. El ejército romano se convirtió sin quererlo en la víctima y el agente transmisor de la pandemia, contaminando las provincias por las que este pasaba y afectando a todas las clases sociales. En el 166 la epidemia había llegado a Roma, siendo su portador el ejército comandado por el propio Lucio Vero, y de allí a finales del 169-170, según Amiano Marcelino, habría alcanzado las Galias y el Rhin. Sin embargo no parece que esta pandemia afectara a Hispania (Gozalbes y Garcia 2007). Dada la diferencia de fechas entre dicha epidemia y los datos que tenemos del siglo III d.C. hemos descartado la peste Antonina como causa originaria del fenómeno observado en el campo Tarragonés. una suposición que solo se verificará con cronologías más precisas y estudios regionales comparativos en otros ámbitos del mediterráneo.

Dejando aparte estas últimas especulaciones realizadas sobre la base de los datos aportados por estos métodos, no sabemos si la población, en principio desplazada, optó por englobar el personal laboral de los propietarios de los fundus supervivientes o pasó a formar parte de los sectores más desfavorecidos de la colonia. Para Tarraco este es un siglo de contrastes pues se realiza una reforma monumental del anfiteatro con la gran inscripción honorífica de Heliogábalo, pero al mismo tiempo el teatro ha quedado abandonado y algunos de sus espacios han sido reaprovechados para construir lugares de hábitat, desmontando y reutilizando el material monumental del recinto (Mar y Ruiz de Arbulo 2011: 532-533) Además, en otros sectores de la ciudad, ya extramuros, las viviendas privadas se abandonan sufriendo algunas de ellas largos periodos de expolio ${ }^{13}$. Es significativo que de una de las canteras extraurbanas de la ciudad deje de extraerse piedra precisamente durante ese periodo (Macias et alii 2007: 151, ficha 612).

\section{BIBLIOGRAFÍA}

Alföldy, G. 1991: Tarraco, Forum 8, Tarragona.

Arrayás, I. 2004: "Morfología histórica del territorium de Tarraco en época tardorepublicana romana (siglos. III-I a.C.)", Dialogues d'Histoire Ancienne, 30 (1), 33-60.

Baxter, M. J, Beardah C. C. y Wright. R.V.S. 1995: "Some archaeological applications of kernel density estimates", Journal of Archaeological Science 24, 347-354.

Bintliff J. L 2009: "Catchements, settlements chambers and demography: case studies and general theory in the Greek landscape from prehistory to early modern times", F. Favory y L. Nuninger (eds.), Archaedyn 7. Millennia of Territorial Dynamics. Settlement pattern, production and trades from Neolithic to Middle Ages, Dijon, 107-117.

Bocquet-Appel J. P, Demars, P., Noiret, L. y Dobrowsky, D. 2005:'Estimates of Upper Palaeolithic meta-population size in Europe from archaeological data", Journal of Archaeological Science 32 (11), 1656-1668.

${ }^{13}$ Por ejemplo en: Alguer 9 (Macias et alii, 138, ficha 551); C Mallorca 20 (Macias et alii, 140, ficha 568); C. Pere Martell 36, 38 y 40 (Macias et alii, 142, fichas 570, 573 y 577); C. Eivissa 16 (Macias et alii, 144, ficha 584); C de Francesc Bastos 24 (Macias et alii, 149, Ficha 602). 
Carreté, J. M., Keay, S. J y Millet M. 1995: “A Roman Provincial Capital and its Hinterland. The Survey of the Territory of Tarragona, Spain, 1985-1990”, Journal of Roman Archaeology, Supplementary series 15.

Chareille. P, Rodier, X. y Zadora-Rio, E. 2005: "La construction des territoires paroissiaux en Touraine: modélisation à l'àide d'un SIG', J. F. Berger, F. Bertoncello, F. Braemer, G. Davtian y M. Gazenbeek (eds.), Temps et spaces de l'homme en societé, analyses et modèles spatiaux en archaeologie, XXV recontre internationales d'archeologie et d'histoire d'Antibes, Antibes, 175-198.

Conolly, J.y Lake, M. 2006: Geographical information Systems in Archaeology, Cambridge.

Crespo, C. 2009: "Los fora de época imperial: los ejemplos alpinos y sardos”, Veleia 26, 287- 295.

Fiz, I. 2010a: "La aplicació dels Sistemes d'Informació Geogràfica (SIG)", M. Prevosti y J. Guitart (eds.), Ager Tarraconensis 2. El poblament, 229-254.

Fiz, I. 2010b: "La visibilitat com a estudi del poblament de l'ager Tarraconensis", M. Prevosti y J. Guitart (eds.), Ager Tarraconensis 2. El poblament, 281-346.

Fiz, I. y Macias. J. M 2007: “L'urbanisme”, J. M. Macias, I. Fiz, L. Piñol y M. Miró (dirs.) Planimetria Arqueologica de Tarragona, 7-40.

Fletcher, M. y Lock, G. 2005: Digging numbers. Elementary statistics for archaeologists, Oxford.

Gorostidi, D., López., J, Prevosti, M y Fiz, I (en prensa): "Propietaris de vil.les de l'ager Tarraconensis (Camp de Tarragona, a la dreta del riu Francolí). Proposta per un catàleg", Actas del congreso. L'ager Tarraconensis. Paisatge, poblament, cultura material i història (Tarragona 27/28 2010).

Gorostidi. D 2010: Ager Tarraconensis 3: Les inscripcions romanes, Tarragona.

Gozalbes. E y Garcia. I 2007: "La primera peste de los Antoninos (165-170). Una epidemia en la Roma Imperial", Asclepio. Revista de la Historia de la Medicina y la Ciencia LIX (1), 7-22.

Gross. P, y Torelli. M. 2007: Storia dell'urbanistica. Il mondo romano, Roma-Bari.

Guitart, J. y Ruestes, C. 2003:'Estudi del paisatge antic de la Cossetània Oriental. Una aplicació arqueològica del GIS Arcview", J. Guitart, J. M. Palet y M. Prevosti (eds), Territoris antics a la Mediterrània i a la Cossetània Oriental. Actes del Simposi Internacional d'Arqueologia del Baix Penedès. El Vendrell, 8-10 novembre 2001, Barcelona, 165-172.

Hare, T. S. 2004: "Using measures of cost distance in the estimation of polity boundaries in the Post- classic Yautepec valley, Mexico", Journal of Archaeological Science 31 (6), 799- 814.

Herzog, I. y Yépez, A. 2010: "Least Cost Kernel Density Estimation and Interpolation-Based Density Analysis applied to survey data", F. J. Melero, P. Cano y J. Rwevelles (eds.) Fusion of cultures, Abstracts of the XXXVIII annual conference on computer applications and quantitative methods in archaeology. CAA 2010 (Granada April 6-9, 2010), 447- 450.

Járrega, R. 2008: "La crisis del segle III a l'àrea compresa entre Tàrraco i Saguntum. Aproximació a partir de les dades arqueològiques", The countryside at the 3rd Century. From Septimus Severus to the Tetrarchy, Studies of the rural world in the roman period 3, 105-139.

López, J., Prevosti, M. y Fiz, I. 2010: “A Study of the Population by Chronological Periods and Typologies", M. Prevosti y J. Guitart (eds.), Ager Tarraconensis 2 El poblament, Tarragona, 372-404.

Löwenborg, D. 2007: "Watersheds as Method for reconstructing regions and territoires in GIS", J. I. Clark y E. Hagemeister (eds.), Digital Discovery. Exploring new frontiers in human heritage. Proceedings of the 34th International Conference on Computer Applications and Quantitative Methods in Archaeology (CAA), Fargo, United States (April 2006), Budapest, 143-149.

Mar, R. y Ruiz de Arbulo, J. 2011: “Tarragona Romana: República y Alto Imperio (218 a.C.- 260 d.C.)", Historia de Tarragona I, 207-538.

Macias, J. M. 2000: "L'urbanisme de Tarraco a partir de les excavacions de l'entorn del Forum de la ciutat", J. Ruiz de Arbulo (ed.), Tarraco 99. Arqueología de una capital provincial romana, Tarragona, 83-106.

Macias, J. M., Fiz, I, Piñol,. L y Miró. M. 2007: Planimetría Arqueológica de Tarraco, Tarragona.

Mar, R., Ruiz de Arbulo, J., Vivó, D. 2010: "El foro de la colonia Tarraco entre la República y el Iimperio", R. Villaescusa y J. Ruiz De Arbulo (eds.), Simulacra Romae II. Rome, les capitales de province (capita provinciarum) et la creation d'un espace commun européen. Une approche archéologique, Memoire 19, 39-70.

Melchor, E. 2009: "Las elites municipales hispanoromanas a fines de la Republica y en el Alto Imperio: ideología y conductas sociopolíticas", J. Andreu, J. Cabrero e I. Rodà (eds.), Hispaniae. Las provincias hispanas en el mundo romano, Tarragona, 391-410.

Moatti, C. 1993 : Archives et partage de la terre dans le monde romain (IIe siècle avant - Ier siècle après J.-C.), Publications de l'École française de Rome 173, Roma. 
Palahí, Ll. 2010: "La romanització al nord-est peninsular i els orígens de la vil-la", Time of Changes. In the beginning of the romanization, Studies of the rural world in the Roman period, 5, 61-108.

Palahí, Ll., Nolla, J. M. y Casas, J. 2008: "El camp en el segle III. Reflexions en veu alta", Time of Changes. In the beginning of the Romanization, Studies of the rural world in the Roman period 3, 11-33.

Palet, J. M. 2003: 'L’organització del paisatge agrari al Penedès i les centuriacions del territori de Tàrraco: estudi arqueomorfològic", J. Guitart, J. M. Palet y M. Prevosti (eds.), Territoris antics a la Mediterrània i a la Cossetània oriental. Actes del Simposi Internacional d'Arqueologia del Baix Penedès, Barcelona, 211-229.

Palet, J. M. 2008: "Formes del paisatge i trames centuriades al Camp de Tarragona: aproximació a l'estructuració del territorio de Tarraco", J. A. Remolá (coord.), El territorio de Tarraco: Vil.les romanes del camp de Tarragona, Forum 13, 49-64.

Palet, J. M, Fiz, I. y Orengo, H. 2010: "Modelación y conceptualización del paisaje romano en el Ager Tarraconensis: Tarraco y la Centuriación del Territorio", C. Corsi y F. Vermeulen (eds.), Changing Landscapes. The impact of Roman towns in the Western Mediterranean. Proceedings of the International Colloquium, Castelo de Vide-Marvão 15th-17th May 2008, 167-184.

Pina Polo, F. 1997: "Las comisiones senatoriales para la reorganización de Hispania (App., Iber. 99-100)", Dialogues d'Histoire Ancienne 23 (2), 83 -104.
Pina Polo, F. 2009: "Hispania y su conquista en los avatares de la república tardía", J. Andreu, J. Cabrero e I. Rodà (ed.), Hispaniae. Las provincias hispanas en el mundo romano, Tarragona, 224-236.

Prevosti, M. y Guitart, J. 2010a: Ager Tarraconensis 1.Aspectes històrics $i$ marc natural, Vols. 1, Tarragona.

Prevosti, M. y Guitart, J. 2010b: Ager Tarraconensis 2. El poblament, Tarragona.

Prieto, A. 2002: "Espacio social y organización territorial de la Hispania romana”, Studia Historica. Historia Antigua 20, 139-170.

Riera, S., Miras, Y., Giralt, S. y Servera, G. 2010: "Evolució del paisatge vegetal al Camp de Tarragona: estudi polinic de la seqüencia sedimentològica procedent de l'aiguamoll de la Sèquia Major (la Pineda,Vilaseca), M. Prevosti y J. Guitart (ed): "Ager Tarraconensis 1, Tarragona. Aspectes històrics i marc natural", Documenta 16-1, Tarragona, 163-172.

Ruiz, A. 2007: "Los Iberos”, F. Gracia (ed.), De Iberia a Hispania, Barcelona, 733-839.

Shennan, S. 2004: Quantifying Archaeology, Edinburgh.

Torelli, M. 1988 : "Della aristocrazie gentilicea a la mascita della pleba”, A. Momigliano y A. Schiavone, Storia de Roma, Turín, 241-261,

Zadora-Rio, E. 2008 : Des paroisses de Touraine aux communes d'Indre-et-Loire. La formation des territoires, Tours. 